\title{
Identification of novel regulatory factor X (RFX) target genes by comparative genomics in Drosophila species
}

\author{
Anne Laurençon ${ }^{* \dagger}$, Raphaëlle Dubruille ${ }^{* \neq}$, Evgeni Efimenko§, \\ Guillaume Grenier $^{* \dagger}$, Ryan Bissett ${ }^{*}+\pi$, Elisabeth Cortier ${ }^{* \dagger}$, Vivien Rolland ${ }^{* \dagger}$, \\ Peter Swoboda§ and Bénédicte Durand ${ }^{* \dagger}$
}

\begin{abstract}
Addresses: *Université de Lyon, Lyon, F-69003, France. 'Université Lyon 1, CNRS, UMR5534, Centre de Génétique Moléculaire et Cellulaire, Villeurbanne, F-69622, France. "University of Massachusetts Medical School, Department of Neurobiology, Worcester, MA 01605, USA. §Karolinska Institute, Department of Biosciences and Nutrition, Södertörn University College, School of Life Sciences, S-14189 Huddinge, Sweden. "University of Glasgow, Glasgow Biomedical Research Centre, Wellcome Centre for Molecular Parasitology and Infection and Immunity, Glasgow G12 8TA, UK.
\end{abstract}

Correspondence: Anne Laurençon. Email: laurencon@cgmc.univ-lyon1.fr

Published: 17 September 2007

Genome Biology 2007, 8:RI 95 (doi:10.1 I86/gb-2007-8-9-rl95)

The electronic version of this article is the complete one and can be found online at http://genomebiology.com/2007/8/9/R/95
Received: 23 July 2007

Revised: 14 September 2007

Accepted: 17 September 2007

(c) 2007 Laurençon et al.; licensee BioMed Central Ltd.

This is an open access article distributed under the terms of the Creative Commons Attribution License (http://creativecommons.org/licenses/by/2.0), which permits unrestricted use, distribution, and reproduction in any medium, provided the original work is properly cited.

\begin{abstract}
Background: Regulatory factor $\mathrm{X}(\mathrm{RFX})$ transcription factors play a key role in ciliary assembly in nematode, Drosophila and mouse. Using the tremendous advantages of comparative genomics in closely related species, we identified novel genes regulated by dRFX in Drosophila.

Results: We first demonstrate that a subset of known ciliary genes in Caenorhabditis elegans and Drosophila are regulated by dRFX and have a conserved RFX binding site (X-box) in their promoters in two highly divergent Drosophila species. We then designed an X-box consensus sequence and carried out a genome wide computer screen to identify novel genes under RFX control. We found 412 genes that share a conserved X-box upstream of the ATG in both species, with 83 genes presenting a more restricted consensus. We analyzed 25 of these 83 genes, 16 of which are indeed RFX target genes. Two of them have never been described as involved in ciliogenesis. In addition, reporter construct expression analysis revealed that three of the identified genes encode proteins specifically localized in ciliated endings of Drosophila sensory neurons.

Conclusion: Our X-box search strategy led to the identification of novel RFX target genes in Drosophila that are involved in sensory ciliogenesis. We also established a highly valuable Drosophila cilia and basal body dataset. These results demonstrate the accuracy of the X-box screen and will be useful for the identification of candidate genes for human ciliopathies, as several human homologs of RFX target genes are known to be involved in diseases, such as Bardet-Biedl syndrome.
\end{abstract}




\section{Background}

Eukaryotic cilia and flagella are present in many types of tissues and organisms and are important for sensory functions, cell motility, molecular transport, and several developmental processes, such as the establishment of left-right asymmetry in vertebrates [1-5]. Several human diseases are known to result from defects in ciliary assembly or function and have recently been designated as ciliopathies [5]. Cilia are welldefined structures consisting of a microtubular axoneme composed of specific proteins that are assembled dynamically in a strict stereotypical pattern (for reviews, see [6,7]). Ciliary assembly depends on intraflagellar transport (IFT) a dynamic process highly conserved in organisms ranging from the green algae Chlamydomonas to mammals (reviewed in $[1,8,9])$. Several studies in various organisms have been instrumental in the identification of genes involved in the assembly and function of the cilium. The proteomic analysis of detergent-extracted ciliary axonemes from cultured human epithelial cells identified 214 proteins [10]. More recently, a biochemical fractionation of Chlamydomonas reinhardtii flagella led to the identification of about 700 proteins, of which 360 had high confidence of truly being involved in flagellar composition [11]. A proteomic analysis of Trypanosoma brucei flagella allowed the identification of 522 proteins [12]. Two remarkable approaches took advantage of the availability of complete genome sequences to identify genes encoding ciliary and flagellar proteins. By comparing the genomes of ciliated versus non-ciliated organisms, Avidor-Reiss et al. [13] and Li et al. [14] selected 187 and 688 genes, respectively, that are specific to ciliated organisms. Stolc et al. [15] used microarray hybridization to analyze induction levels of all $C$. reinhardtii genes after deflagellation. They identified 220 genes that are induced at least two-fold and, therefore, are likely to be involved in the assembly or function of cilia and flagella.

Much less is known about the regulatory pathways that control the expression of ciliary components or direct the differentiation of ciliated cells. The transcription factor FoxJ1 appears to govern the differentiation of ciliated cells in vertebrates, but so far, only one gene has been shown to be directly regulated by FoxJ1 [16]. The transcription factor HNF1- $\beta$ has also been shown to regulate several genes involved in ciliogenesis in the kidney [17]. Most importantly, regulatory factor $\mathrm{X}$ (RFX) transcription factors play a key role in regulating genes involved in ciliogenesis. RFX transcription factors are conserved in a wide range of species, including Saccharomyces cerevisiae, Caenorhabditis elegans, Drosophila melanogaster and mammals. They share a characteristic DNAbinding domain of the winged-helix DNA binding family and bind to an X-box motif, an imperfect inverted repeat with variable spacing between the repeats $[18,19]$. Whereas only one $R f x$ gene is described in yeast and $C$. elegans, two $R f x$ genes are present in the Drosophila genome and five in mammals [20]. Major clues on RFX functions in metazoans have been obtained from work on invertebrates. $d a f-19$, the sole $R f x$ gene in C. elegans, is a key regulator of ciliogenesis [21]. $d R f x$ in Drosophila is expressed in ciliated cells and is necessary for ciliated sensory neuron differentiation: all sensory neurons are present but cilia are missing at the dendritic tips [22,23]. In mouse, we have shown that RFX function in ciliogenesis is conserved. Indeed, $R f x_{3}$ controls the growth of mouse embryonic node cilia [24] and $R f x_{3}$ loss-of-function leads to hydrocephalus with differentiation defects of ciliated ependymal cells of the choroid plexus and subcommisural organ [25]. Moreover, $R f_{3} 3$ mutant mice show insulin secretion failure and impaired glucose tolerance correlated with primary ciliary growth defects on islet cells [26]. In zebrafish, Rfx2 is expressed specifically in multiciliated cells of the pronephros and loss of $R f x 2$ leads to cyst formation and loss of multicilia [27]. The function of the other RFX proteins has yet to be linked to ciliogenesis. $R f x_{5}$, the most divergent mammalian member, regulates major histocompatibility class II gene expression and mutations in it are responsible for the bare lymphocyte syndrome [28]. $R f x 4$ has been implicated in dorsal patterning of brain development in mice and may participate in circadian rhythm regulation in humans [29-32].

Because RFX function in ciliogenesis appears conserved from C. elegans to mammals, X-box promoter motif sequences can guide the search for ciliary genes. Indeed, genome wide searches for genes controlled by DAF-19 in C. elegans have identified many genes involved in ciliogenesis [14,21,33-38]. Genomic X-box searches thus comprise a key method to identify genes involved in ciliary development. We show here that ciliogenic RFX regulatory cascades are well conserved between $D$. melanogaster and $C$. elegans and identify a first set of 14 RFX target genes. In particular, we show that all known Drosophila homologs of genes defective in human Bardet-Biedl syndrome (BBS), a human ciliopathy with complex phenotypes, are controlled by dRFX. Moreover, by using comparative genomic screens we show that genes under dRFX control in D. melanogaster share conserved X-boxes with another divergent Drosophila species, D. pseudoobscura. Applied to the whole genome of both species, our comparative approach led to the identification of at least 11 novel RFX target genes. In vivo reporter assay studies for three of them confirmed their involvement in ciliary structure or function in Drosophila, thus illustrating the accuracy of our screen. In addition, we have established a highly confident Drosophila cilia and basal body (DCBB) gene list and highlight several genes as novel candidates for ciliogenesis. Our data are of particular importance for further genetic and genomic studies in the field of ciliogenesis and, consequently, for identifying genes involved in human ciliopathies.

\section{Results \\ Homologs of C. elegans DAF- 9 target genes are regulated by dRFX in Drosophila}

Our previous work has shown that RFX transcription factors share a common function in ciliogenesis in worm and fly 
Table I

\begin{tabular}{|c|c|c|c|c|c|}
\hline D. melanogaster gene ID (name) & $\begin{array}{l}\text { Homologs in } \\
\text { vertebrates or } \\
\text { Chlamydomonas }\end{array}$ & Fold variation & $\begin{array}{l}\text { Ciliary } \\
\text { type } \\
\text { [13] }\end{array}$ & C. elegans gene ID (name) & $\begin{array}{l}\text { DAF-19 control } \\
\text { in C. elegans }\end{array}$ \\
\hline \multicolumn{6}{|l|}{ Downregulated $>2$ fold } \\
\hline CGII26 & BBS5 & $95.2^{*}$ & $C_{p}$ & ROIHI0.6 (bbs-5) & All [14] \\
\hline CG3769 & D2LICILIC3 & $63^{*}$ & - & F02D8.3 $(x b x-I)$ & All $[21,35]$ \\
\hline CG4525 & Novel & $223.6^{*}$ & $C_{p}$ & C27H5.7 (dyf-I3) & All [37] \\
\hline CG8853 & IFT55/hippi & $21.8^{*}$ & $C_{p}$ & F59C6.7 (che-13) & All [33] \\
\hline CG9333 (oseg5) & WDR56 & $4.4^{*}$ & $C_{p}$ & F38GI.I (che-2) & All [2I] \\
\hline CG9595 (osm-6) & NDG5 & $22.2^{*}$ & $C_{p}$ & R3I.3 (osm-6) & All [2I] \\
\hline CGI2548 (nompB) & TG737 & $12.7[92]^{*}$ & $C_{p}$ & Y4lg9a.I (osm-5) & All [34] \\
\hline CGI369I (BBS8) & BBS8 & $2.7^{*}$ & $C_{p}$ & T25FI0.5 (bbs-8) & All [36] \\
\hline CGI3809 (oseg2) & IFTI 72/wim & $9.7 *_{\text {in vivo }}$ & $C_{p}$ & T27BI.I (osm-l) & All [2I] \\
\hline CGI4825 (BBSI) & BBSI & $211 *$ & $C_{p}$ & YI05E8A.5 (bbs-l) & All $[36,37]$ \\
\hline CGI5666 & BBS9 & $20^{*}$ & - & C48B6.8 & All [37] \\
\hline CGI7599 & QILIN & $29.5^{*}$ & $C_{p}$ & C04C3.5 (dyf-3) & All [93] \\
\hline CG9398 (Tulp) & Tubby & $3.2 \ddagger$ & - & FIOB5.4 (tub-l) & Subset [36] \\
\hline CG3259 & Traßßip I/MIP-T3 & In vivo & $C_{p}$ & $\mathrm{C} 02 \mathrm{H} 7 . \mathrm{I}$ & ND \\
\hline CG5I42 & Novel & $16.4^{*}$ & $C_{p}$ & F54CI.5a (dyf-I) & ND \\
\hline CG7735 & BBS3 & $40.9 *$ & $C_{p}$ & C38D4.8 (arl-6) & ND \\
\hline CG9227 (tectonic) & tectonic & In vivo & $C_{p}$ & Y38F2AL.2 & ND \\
\hline CGI4367 & Novel & $10.2+$ & $C_{p}$ & YI08G3AL.3 & ND \\
\hline CGI4870 & B9 domain & $3.1 *$ & $C_{p}$ & K03E6.4 & ND \\
\hline CGI5I6I & IFT46 & $207.1^{*}$ & $C_{p}$ & F46F6.4 (dyf-6) & ND \\
\hline CGI863I & Novel & $14.2^{*}$ & $C_{p}$ & K07G5.3 & ND \\
\hline CGI3232 (BBS4) & BBS4 & $17^{*}$ & - & - & - \\
\hline \multicolumn{6}{|l|}{ Downregulated $<2$-fold } \\
\hline CG3044I & IFT20 & $2 \ddagger$ & $C_{p}$ & YIIOA7A.20 & All [37] \\
\hline CGI399-PB & LRRCI6 & $1.8^{\dagger}$ & $C_{p}$ & K07G5.I & ND \\
\hline CGII048 & Rib74 & $1.5^{\dagger}$ & $C_{p}$ & Y49AIOA.I & ND \\
\hline CGI3I78 & Novel & $1.7 \dagger$ & $C_{p}$ & RIOF2.5 & ND \\
\hline \multicolumn{6}{|l|}{ Overexpressed } \\
\hline CG2006 & DtwdI & $0.5^{*}$ & $C_{p}$ & Y53CI2A.3 & \\
\hline \multicolumn{6}{|l|}{ Invariant in Drosophila } \\
\hline CG5359 & Tctex & I & & DI009.5 (xbx-2/dylt-2) & All [36] \\
\hline CGII237 (oseg6) & $w d r 19$ & I & $C_{p}$ & ZK520.3 (dyf-2) & Subset [94] \\
\hline CG6504 (amo) & PKD2 & 0.9 & & Y73F8A.I (pkd-2) & Subset [95] \\
\hline CG97I0 (nudC) & NudC & 0.7 & & F53A2.4 (nud-I) & Subset [36] \\
\hline CGII838 (oseg3) & IFTI 40 & 1.4 & $C_{p}$ & C27A7.4 (che-II) & Subset [36] \\
\hline CG2069 (oseg4) & WDR35 & 1 & $C_{p}$ & C54G7.4 (ifta-l) & ND \\
\hline CG6560 & $a r l 3$ & 1 & $C_{p}$ & FI9H8.3 (arl-3) & ND \\
\hline CG7I6I (osegl) & IFTI22 & 1.4 in vivo & $C_{p}$ & F23B2.4 (daf-10/osm-4) & ND \\
\hline CGI0642 (Klp64D) & KIF3A & 1.1 & $C_{p}$ & Y50D7A.6 (klp-20) & ND \\
\hline CGII755 & Novel & 1.1 & $C_{p}$ & D2089.3 & ND \\
\hline CGII759 (Кар3) & KIFAP3 & 1 & $C_{p}$ & F08F8.3 (kap-l) & ND \\
\hline CG5I95 & L domain like & 1.2 & $C_{p}$ & - & - \\
\hline CG31249 & Novel & 0.9 & $C_{p}$ & - & - \\
\hline
\end{tabular}


Table I (Continued)

\begin{tabular}{|c|c|c|c|c|c|}
\hline CG32392 & $r s h l 2$ & $\mathrm{I} . \mathrm{I}$ & $C_{p}$ & - & - \\
\hline \multicolumn{6}{|c|}{ Not determined in Drosophila } \\
\hline CG3798 (Nmdal) & & ND & & F40F9.I $(x b x-6)$ & Subset $[36]$ \\
\hline CG93IO (Hnf4) & HNF4A/G & ND & & TI9A5.4 (nhr-44) & Subset [36] \\
\hline CGI7228 (pros) & PROXI/2 & ND & & KI2H4.I (ceh-26) & Subset [95] \\
\hline \multicolumn{6}{|c|}{ Not conserved in Drosophila } \\
\hline- & & - & & Y75B8A. 12 (osm-/ 2/bbs-7) & All [36] \\
\hline- & & - & & F20DI2.3 (bbs-2) & All [36] \\
\hline- & & - & & M28.7 (nph-l) & All [96] \\
\hline- & & - & & $\mathrm{RI} 3 \mathrm{H} 4 . \mathrm{I}(n p h-4)$ & All [96] \\
\hline- & & - & & C47E8.6 & All [37] \\
\hline- & & - & & ZK328.7a & All [37] \\
\hline- & & - & & YI02E9.I (odr-4) & Subset $[36]$ \\
\hline- & & - & & C23H5.3 (xbx-4) & Subset $[36]$ \\
\hline- & & - & & M04D8.6 (xbx-3) & Subset $[36]$ \\
\hline- & & - & & T24AII.2(xbx-5) & Subset [36] \\
\hline- & & - & & RI48.I (xbx-7) & Subset [36] \\
\hline- & & - & & D2005.2 (n/p-8) & Subset [95] \\
\hline
\end{tabular}

$*_{p}$ value $<0.001 ;$ †0.00I $<p$ value $<0.05 ; \neq 0.05<p$ value $<0.08$. Dashes indicate not applicable. Cp, compartmentalized ciliogenesis; ND, not determined.

$[21,23]$. We thus inferred that an identical set of genes would be regulated by DAF-19 in C. elegans and dRFX in D. melanogaster. Indeed, among more than 20 previously identified DAF-19 targets expressed in all ciliated sensory neurons of $C$. elegans [21,36-38], we show that a majority of the homologous genes in fly are down regulated in $d R f x$ mutants (Table 1). Regulation of gene expression was tested by real-time PCR based on RNA extracted from 40-hour old pupae thoraxes and legs. At this stage, dendrites and cilia have just differentiated. Moreover, the levels of expression of ciliary genes osm6 and nompB, relative to the housekeeping gene TBP (TATA Binding Protein) or the pan-neural gene elav during pupae development, is at a maximum starting at 40 hours after puparium formation (data not shown). As shown in Table 1, 14 of 19 DAF-19 regulated genes for which a homologous gene can be found in Drosophila are also regulated by dRFX. Only one gene (CG5359/D1009.5/xbx-2/dylt-2) regulated by DAF-19 in all ciliated sensory neurons in C. elegans does not seem to be under dRFX regulation in Drosophila. Among all the $C$. elegans genes expressed and regulated by DAF-19 in a subset of ciliated sensory neurons, only CG9398/tulp appears to be under dRFX control in Drosophila. All the others, such as oseg3, NudC or amo, do not appear to be regulated by dRFX in our assay conditions. However, we cannot exclude that these genes are under dRFX regulation in a small subset of ciliated sensory neurons and, thus, that variations of their expression cannot be detected by real time RT-PCR of RNA preparations of pupae thoraxes and legs. Remarkably, genes that are involved in BBS and conserved in both organisms are regulated by RFX proteins. We quantified the expression of $C G 13232 / B B S 4$ in Drosophila, the only BBS gene that is not found in the $C$. elegans genome, and show that it is also down regulated 17-fold in a $d R f x$ deficient background. Most of the other genes regulated by dRFX are involved in IFT. This transport is led by two types of molecular motors, anterograde kinesins and retrograde dyneins, that carry particles that can be biochemically fractionated as A and B complexes [1]. dRFX regulates genes encoding B complex components, but not A complex components.

\section{Genes specific to compartmentalized ciliogenesis are regulated by dRFX in Drosophila}

Interestingly, most of the genes regulated by dRFX also fall in the list of genes for compartmentalized ciliogenesis ( $\mathrm{Cp}$ ciliary type, Table 1) defined by the work of Avidor-Reiss et al. (Table 1) [13]. This group of genes is found only in genomes of species showing compartmentalized cilia biogenesis, but neither in the genomes of non-ciliated organisms nor in Plasmodium falciparum, which uses cytosolic cilia biogenesis. We thus tested the expression of almost all the genes described in the $\mathrm{Cp}$ category in control and $d R f x$ deficient Drosophila. Among the $34 \mathrm{Cp}$ ciliary genes tested by real-time PCR, 18 were down regulated more than 2-fold in a $d R f x$ mutant background, 4 were significantly reduced between 1.5 - and 2-fold 
and one was significantly over expressed. Eleven genes did not show significant expression variations between control and mutant background (Table 1).

In order to demonstrate the accuracy of our quantification procedure, we performed in vivo observations of reporter constructs of some of the genes in wild-type and $d R f x$ deficient backgrounds (Figure 1). As previously published, sensory neuron ciliary endings are missing in a $d R f x$ deficient background [23]. As observed in the cell body or remaining dendrite, the expression of osm-1 is totally shut down in the $d R f x$ deficient background, whereas the expression of oseg 1 is not affected (Figure 1), in agreement with real-time RT-PCR results. Interestingly, CG3259 and CG9227 cDNAs were hardly detectable by real-time PCR and, thus, difficult to quantify. However, in vivo observations of reporter constructs in wild-type and $d R f x$ mutant backgrounds show a complete absence of expression of these two genes in the mutant background (Figure 1).

In summary, we show that RFX target genes are mainly conserved between $C$. elegans and D. melanogaster. Our functional comparative approach between both organisms combined with the work of Avidor-Reiss et al. in Drosophila allowed us to identify 27 genes that are regulated by dRFX in Drosophila. A majority of them are shown to be involved in ciliogenesis.

\section{X-box conservation between $D$. melanogaster and $D$. pseudoobscura}

As previously described [13,14,21,36-39], the X-box promoter motif has been used successfully to screen for genes involved in ciliogenesis. As shown above, this first set of X-box gene data in Drosophila is thus a key to better understand the link between X-box sequences and dRFX transcriptional control in Drosophila. We looked for X-boxes in the promoters of dRFX target genes. We searched for X-boxes up to $3 \mathrm{~kb}$ upstream of the ATG for each of them, with the most degenerated X-box consensus deduced to date from known RFX protein binding sites (RYYNYY N1-3 RRNRAC). We could identify several X-boxes for each gene (Table 2, columns 2 and 3). However, known negative control genes also presented X-boxes at the same frequency and no particular constraint on the consensus seemed to correlate with one set of genes. Therefore, the presence for one gene of an X-box upstream of its ATG is not predictive of dRFX-dependent expression. We thus turned to the $D$. pseudoobscura genome. The two Drosophila species' most recent common ancestor occurred 40-60 million years ago. The average identity of coding sequence between D. melanogaster and D. pseudoobscura at the nucleotide level is $70 \%$ for the first and second bases of codons, and $49 \%$ for the wobble base. Intron sequences are 40\% identical, untranslated regions 45-50\%, and DNA protein binding sites extracted from the literature have been estimated to an average of 63\% [40]. Moreover, detailed comparison of both Drosophila genomes showed that 50-70\% of known DNA binding sites reside in conserved sequence blocks in the genomes, called conserved regulatory elements (CREs), whereas the overall conservation of the cisregulatory regions is low [41-43].

We thus looked for D. pseudoobscura homologs of either dRFX positively regulated or invariant genes and for X-boxes up to $3 \mathrm{~kb}$ upstream of the ATG. Interestingly, 70\% of conserved dRFX target genes present a conserved X-box in both species (Table 2), whereas only $23 \%$ of negative control genes present the same characteristic. Even more precisely, while the sequence and the location of X-boxes for dRFX target genes are conserved, this is not the case for negative control genes. Interestingly, palindromic $\mathrm{X}$-boxes are significantly over-represented compared to non-palindromic X-box sequences in dRFX regulated genes in the two species.

We also looked for overall sequence conservation around the selected X-boxes by Vista promoter sequence comparison between the two Drosophila species. The percentage of identities was quantified either on 100 bp or 25 bp windows surrounding the X-boxes (Figure 2, Table 2) and block conservation was considered positive if identities were over $50 \%$. As shown in Table 2, sequences around the X-boxes are generally not well conserved. Two representative examples are depicted in Figure 2. For the CG9595/osm-6 gene, one of the two conserved X-boxes falls into an overall conserved 100 bp block, whereas the other one does not. For CG8853/che13, the X-box falls into a poorly conserved region. These results are in agreement with previously published data showing that sequence block conservation alone cannot discriminate regulatory regions, but that binding site clusters present in multiple species more likely discriminate active and inactive clusters [43].

\section{Screening Drosophila species' genomes for dRFX regulated genes}

The presence of a conserved X-box upstream of genes in both D. melanogaster and D. pseudoobscura is thus a good prognostic factor to predict novel dRFX target genes. We thus screened the genome of both Drosophila species for the presence of X-boxes. We searched for all possible matches to a defined motif sequence using a Perl based algorithm [36]. The most degenerated consensus RYYNYY N1-3 RRNRAC found 50,000 hits throughout the entire genome of D. melanogaster and, therefore, could not be used within our experimental framework. We selected five different more restricted consensus motifs that cover X-boxes of the entire set of known target genes at the time (see Materials and methods). Four (RYYVYY N1-3 RRHRAC, GYTNYY N1-3 RRNRAC, GYTDYY N1-3 RRNRAC, GYTRYY N1-3 RRHRAC) were searched in a $1 \mathrm{~kb}$ window upstream of the ATG, and the less degenerated one, RTNRCC N1-3 RGYAAC, in a $3 \mathrm{~kb}$ window.

Under these conditions, 4,726 non-redundant genes in $D$. melanogaster and 3,848 in D. pseudoobscura with an X-box 


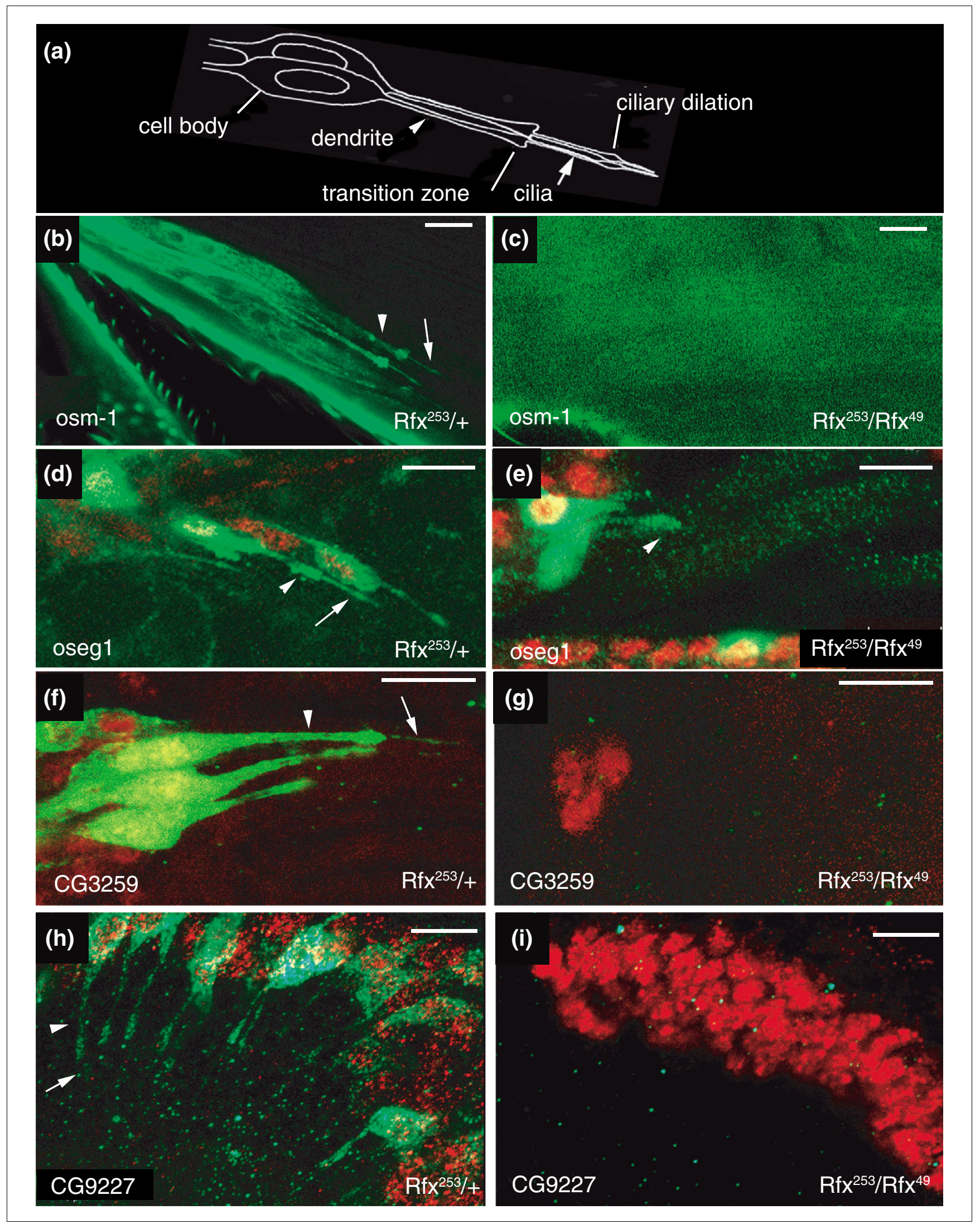

Figure I (see legend on next page) 
Figure I (see previous page)

In vivo observations of reporter constructs in control or $d R f x$-deficient Drosophila. (a) Schematic of two typical chordotonal organs of the Drosophila leg or antenna. The different segments of the dendrite and of the ciliated ending are shown. Sensory neurons have a single cilium (arrow) extending from their dendrite (arrowhead). (b) Live confocal image of GFP driven expression of osm-I transgene in a control femur. (c) GFP expression is totally shut down in a $d R f x$ mutant background. (d-i) Confocal imaging of chordotonal neurons labeled with anti-ELAV (red) and anti-GFP (green). oseg I-GFP expression in (d) control flies and (e) a dRfx mutant background. Note that oseg I-GFP expression is not affected in the mutant background. CG3259-GFP expression in (f) control flies and $(\mathrm{g}) d R f x$ mutant flies. Reporter construct expression is totally shut down in the mutant background. Johnston's organs from antennae of adult flies carrying CG9227-GFP transgenes in (h) control and (i) $d R f x$ mutant pupae. Note the absence of expression in the mutant background. Scale bar = $10 \mu \mathrm{m}$.

upstream of the start codon were selected. Based on a best hit reciprocal search between the two coding sequence (CDS) lists, we identified 1,462 homologous genes having an X-box in their 5 ' region in both species. This first set of 1,462 genes was further restricted by selecting only genes that share an Xbox with no more than 4 bases different (out of the 12 nucleotides recognized by the protein on either side of the spacer) between each species and in a conserved position upstream of the ATG (500 bp difference at most). The list was thus restricted to a subset of 412 genes (Additional data file 1). An even more restricted subset of genes was selected using the Xbox motif GYTRYY N1-3 RRHRAC, which was found upstream of most known target RFX genes at the beginning of this work, leading to a list of 83 genes (Table 3). Indeed, among the identified dRFX target genes for which a conserved X box was found in both Drosophila species (Table 2), the highest percentage of target genes (50\%, 8 out of 16) was found in this list of 83 genes. The remaining $50 \%$ of known RFX target genes (Table 2) were not selected by the X-box screen and thus represent false negatives (see Discussion for a comprehensive analysis).

\section{$X$-box genes and ciliogenesis}

In order to check for enrichment of genes involved in ciliogenesis, we compared our three X-box gene lists to previously published lists of genes potentially involved in cilium or centrosome composition. We first identified the Drosophila homologs for the full set of previously published genes from various organisms from several studies. These include comparative genomic studies of species that have cilia versus species that do not and proteomic analyses of human cilia and centrosome, Chlamydomonas flagellar or basal body and Trypanosoma brucei proteomes [10-14,44,45]. This set also includes recent genome-wide transcriptional analysis of gene expression during flagellar regeneration in Chlamydomonas or identified by SAGE analysis of ciliated neurons combined with X-box searches in C. elegans [15,36,37]. The full set of Drosophila homologs that we found for all studies combined is listed as the DCBB gene set (Additional data file 2).

Interestingly, comparing our set of 1,462 Drosophila X-box candidate genes with the DCBB dataset shows that our list is slightly enriched in DCBB genes. Whereas $5 \%$ of the D. melanogaster genome is in the DCBB dataset, our 412 and the 83 $\mathrm{X}$-box gene candidate datasets appear to be highly enriched in
DCBB genes (11\% and $22 \%$, respectively), suggesting that the $\mathrm{X}$-box conservation is a good marker for genes potentially involved in ciliogenesis (Table 4).

The full set of genes with a putative function in ciliogenesis has also been summarized in parallel in two independent databases called the Ciliary proteome and Ciliome databases [46-49]. Surprisingly, when we compared the two published databases with the DCBB dataset that we established for Drosophila using similar comparative methods (see Materials and methods and Additional data file 2), we observed large discrepancies between all three datasets (illustrated in Figure 3 and Additional data file 3). There are some differences between the three studies with regard to the initial published sets of genes that were included in the database. The major difference resides in which data are included from the work of Blacque et al. [37]. The Ciliome database [47] includes the complete SAGE dataset from Table S1 in [37], whereas our DCBB dataset includes only data from Table 1 from Blacque et al. (2005), which contains part of the SAGE data combined with an X-box search. The ciliary proteome database [46] includes data from Table $\mathrm{S} 4$ of the Blacque et al. study [37], which reports the list of putative X-box genes in the nematode. These differences could account for the high number of genes exclusively represented in the Ciliome database [47] but cannot account for all the discrepancies between our DCBB dataset and the Ciliary proteome database [46] (Additional data file 3). Very likely, the differences observed between all three studies illustrate the problems inherent in automatically processing published tables and gene lists that are then used to compile homologous genes from several different organisms. Another major explanation for the observed discrepancies resides in the order BLAST searches were performed to create each database. For example, the Ciliary proteome database [46] was obtained by looking first for human homologs for each study, and then for the Drosophila ones (unless Drosophila was the starting study). In our DCBB dataset, we have looked for Drosophila homologs, which were then compared to other datasets. Hence, genes that do not have an ortholog in Drosophila or in human are lost in the respective studies.

However, we show that our lists of 412 and 83 X-box genes are enriched in genes involved in ciliogenesis, whatever database is considered (Table 3, Additional data file 1). Thus, our 
Table 2

X-box comparisons in promoters of dRFX regulated genes, between Drosophila melanogaster and Drosophila pseudoobscura

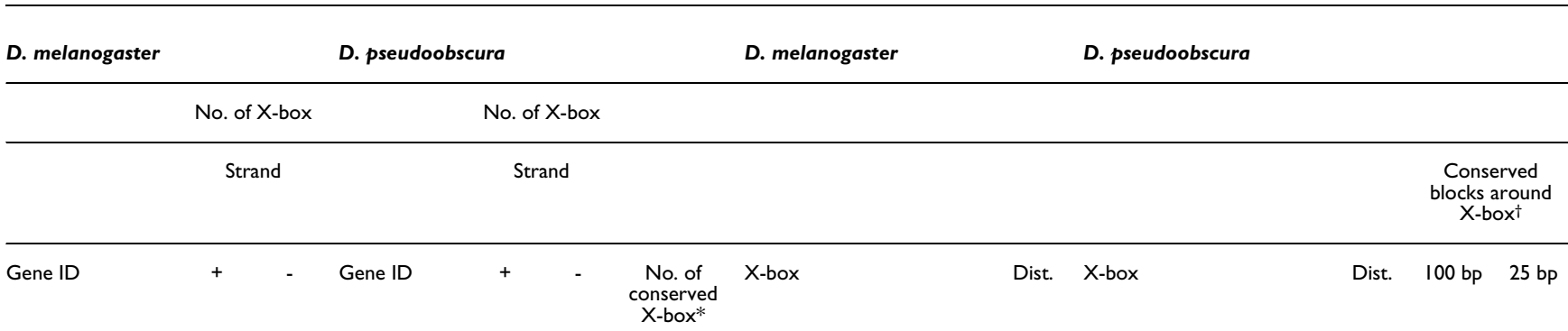

Genes down regulated in $d R f x$ mutant

$\begin{array}{lllllll}\text { CGII26-PA } & 2 & 4 & \text { GAI0872-PA } & 2 & \text { I } & \text { I } \\ \text { CG3259-PA } & 4 & 4 & \text { GAI70II-PA } & 2 & 2 & \text { I } \\ \text { CG3769-PA } & 2 & 3 & \text { GAI7674-PA } & 4 & 3 & \text { I } \\ \text { CG4525-PA } & 2 & 3 & \text { GA18233-PA } & \text { I } & \text { I } & \text { I } \\ \text { CG8853-PA } & 2 & 4 & \text { GA21369-PA } & \text { I } & 5 & \text { I } \\ \text { CG9227-PA } & 5 & \text { I } & \text { GA21627-PA } & 3 & \text { I } & \text { I } \\ \text { CG9595-PA } & 3 & 2 & \text { GA2190I-PA } & 3 & 2 & \text { I }+2\end{array}$

\begin{tabular}{|c|c|c|c|c|c|c|}
\hline CGI2548-PA & 7 & 2 & GAII 690-PA & 2 & 4 & 2 \\
\hline CGI3I78-PA & 4 & 2 & GAI2098-PA & 5 & 4 & I \\
\hline CGI3809-PA & 1 & 2 & GAI2544-PA & 2 & 2 & If \\
\hline CGI5I6I-PA & 2 & 1 & GAI354I-PA & 4 & 2 & $2 \ddagger$ \\
\hline CG3044I-PA & 1 & 3 & GAI5848-PA & 3 & I & $I \ddagger$ \\
\hline CGI7599-PA & 1 & 5 & GAI4573-PA & I & I & If \\
\hline CGI863I-PA & 2 & 2 & GAI5024-PA & 6 & I & I \\
\hline CGI5666-PA & 3 & 3 & GAI388I-PA & 2 & 3 & If \\
\hline CGI4870-PA & 1 & 1 & GAI33I0-PA & I & I & If \\
\hline CGI399-PB & 3 & 3 & GAI2678-PA & 3 & 0 & 0 \\
\hline CG5I42-PA & 3 & 2 & GAI8687-PA & 2 & I & 0 \\
\hline CG9333-PA & 4 & 1 & GA21709-PA & 2 & I & 0 \\
\hline CG9398-PA & 2 & 2 & GA2I760-PA & 3 & 3 & 0 \\
\hline CGII048-PA & 1 & 0 & GAI0726-PA & 5 & 4 & 0 \\
\hline CGI369I-PA & 1 & 1 & GAI2462-PA & I & I & 0 \\
\hline CGI4367-PA & 0 & 3 & GAI2937-PA & 3 & 3 & 0 \\
\hline
\end{tabular}

Invariantly expressed genes in $\mathrm{dRfx}$ mutant

$\begin{array}{lllllll}\text { CG5I95-PA } & 0 & 4 & \text { GAI8727-PA } & 3 & 4 & 0 \\ \text { CG5359-PA } & 4 & 4 & \text { GAI70II-PA } & 2 & \text { I } & \text { I } \\ \text { CG6504-PA } & 3 & 0 & \text { GAI9567-PA } & 6 & 4 & 0 \\ \text { CG6560-PA } & 3 & 0 & \text { GAI9685-PA } & 0 & 2 & 0 \\ \text { CG7I6I-PA } & 2 & 5 & \text { GA20I45-PA } & 0 & 3 & 0 \\ \text { CG97I0-PA } & 2 & 0 & \text { GA2I982-PA } & \text { I } & 0 & 0 \\ \text { CGI0642-PA } & 4 & 2 & \text { GAI0463-PA } & \text { I } & 3 & \text { I } \\ \text { CGII755-PA } & \text { I } & 5 & \text { GAIII76-PA } & 2 & \text { I } & \text { I } \\ \text { CGII237-PA } & 3 & 2 & \text { GAI0856-PA } & \text { I } & 2 & 0 \\ \text { CGII759-PA } & \text { I } & 3 & \text { GAIII78-PA } & 3 & 2 & 0 \\ \text { CGII838-PA } & 3 & 0 & \text { GAII225-PA } & 3 & 4 & 0 \\ \text { CG31249-PA } & \text { I } & \text { I } & \text { GAI6123-PA } & \text { I } & \text { I } & 0 \\ \text { CG32392-PA } & 4 & 2 & \text { GAI6867-PA } & 3 & 3 & 0\end{array}$

GTTGCC T AGCAAC 66 GTTGCCTAGCAAC $\quad 91 \quad-\quad+$ GTTGCC AG GACAAC $81 \quad$ GTTGTCAG GACAAC $96 \quad$ - + GTTGCT AGT AGCAAC 7I GTTGCC AG GACAAC $56 \quad$ - + GTTGCCA AGCGAC 134 GTTGCCAAGCGAC $239+$ GTTACC TT GGCGAC 87 GTTACCAT GGAAAC $110 \quad-+$ GTTACT TT GACAAC $\quad 119 \quad$ GTTGCCAG AGCAAC $146 \quad+\quad+$ GTTGCC G GGAAC $\quad 126$ GTTGTCCG GGCAAC $|4|++$ ATTTTT GTT AGCAAC 264 ACTTTT GC AAAAAC $699 \quad-\quad+$ $\begin{array}{llllll}\text { GCTGTT ACA AGAGAC } & 2,969 & \text { GCTGCT GCA GGAAAC } & 2,671 & \text { NA } & \text { NA }\end{array}$ ATCACC AA GGCAAC $\quad 2,335 \quad$ ATCACC TT GGAAAC $868 \quad$ $\begin{array}{llllll}\text { GCCTTT C GGAGAC } & 2,833 & \text { GCCGCT T GATGAC } & 2,638 & \text { - }\end{array}$

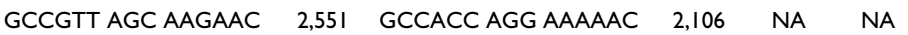
GTTGCC AC AACAAC 123 GTTGCC AC AACAAC $103+$ GTTGTC AG GACGAC 321 GTTGTC AA GACAAC 311 - GTTGTC AG GACGAC $321 \quad$ GTTTTT GCA GGCAAC $391 \quad$ GTTGTC AAT AGCAAC $\quad 60 \quad$ GTTGTC TGT GACAAC $122 \quad$ GTTACC T AGCAAC $\quad$ I4I GTTGCCTGGCAAC 206 GTTGCC CAT GGCAAC 2,731 GTTGCC GTT AGCAAC $\quad 2,633 \quad$ - + GTTGCC AA GGCAAC $88 \quad$ GTGGCC AT GGCAAC $\$ 5 \quad$ GTCTCC CG GGCAAC $\quad-22$ GTATCC TG GGCAAC $\$-6 \quad+$

*RYYNYYN[I-3]RRNRAC. Dist., distance upstream of the ATG. TThe percentage of identities of either 100 bp or 25 bp sequence blocks around the $X$-box was estimated by Vista comparison as positive $(+)$ if identities are above $50 \%$, or negative $(-)$ if below $50 \%$. $\neq$ Palindrome. $\$$ The $X$-box fits the more degenerated consensusRYNNYYN[I-3]RRNRAC. NA, not applicable because of nearby coding sequence environment or gap in the sequences. 


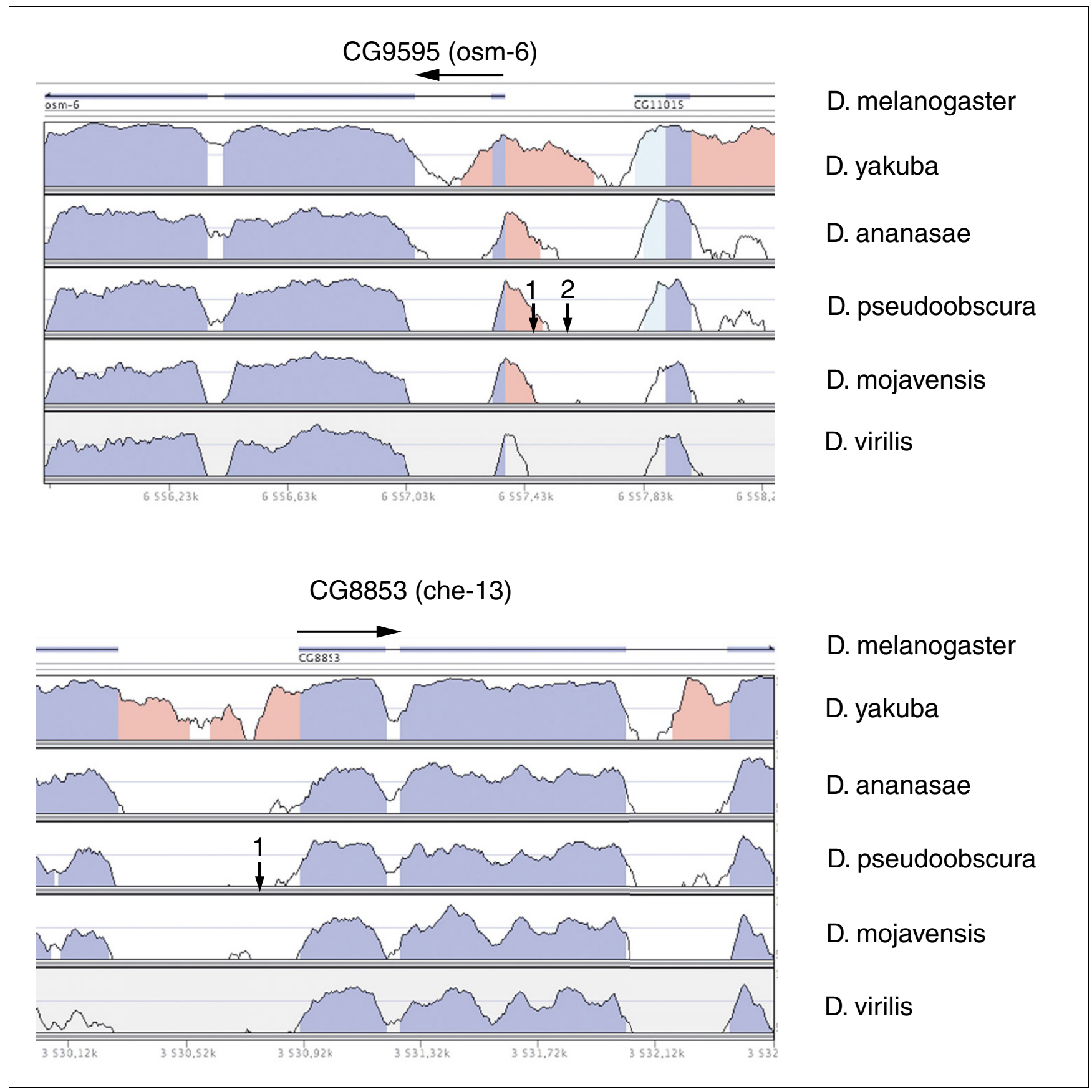

Figure 2

Promoter comparisons between Drosophila species. Sequence identities (from 50-100\%) between different Drosophila species ranging from D. melanogaster to the most distant $D$. virilis as calculated and presented in the VISTA interface [9I] for two dRfx target genes, CG9595 (osm-6/NDG5) and CG8853 (IFT55/ che-I/Hippi). Coding sequences are depicted in dark blue, untranslated regions are in light blue and other conserved regions in pink. Gene orientation is shown by a horizontal arrow. The location of conserved X-boxes for each gene is indicated by numbered vertical arrows. Note that one conserved X-box for osm- 6 is in a conserved block of sequence, while others (osm-6 and che-/3) are not. 
Table 3

Eighty-three genes selected for a conserved $X$-box between $D$. melanogaster and $D$. pseudoobscura

\begin{tabular}{|c|c|c|c|c|c|c|c|c|c|c|c|c|c|c|c|c|c|c|c|}
\hline \multirow[b]{2}{*}{$\begin{array}{l}\text { CG\# release } \\
3.2 .1\end{array}$} & \multirow[b]{2}{*}{$\begin{array}{l}\text { Rfx target fold } \\
\text { reduction }\end{array}$} & \multirow[b]{2}{*}{$\begin{array}{l}\text { Flybase gene } \\
\text { name }\end{array}$} & \multirow[b]{2}{*}{$\begin{array}{l}\text { Known protein } \\
\text { function }\end{array}$} & \multirow[b]{2}{*}{$\begin{array}{l}\text { Annotated predictive } \\
\text { function }\end{array}$} & \multirow[b]{2}{*}{ [12] } & & & & & & & rce [re & erence] & & & & & & \\
\hline & & & & & & {$[11]$} & {$[14]$} & [13] & {$[10]$} & {$[15]$} & [36] & [37] & [45] & [44] & [59] & DCBB & $\begin{array}{l}\text { Ciliome } \\
\text { database }\end{array}$ & $\begin{array}{c}\text { Ciliary } \\
\text { proteome } \\
\text { database }\end{array}$ & Human homologs \\
\hline 1126 & 95 & CGI/26 & BBS5 & & & & $v$ & $v$ & & & $v$ & $v$ & & & & $v$ & $v$ & $v$ & NP_689597 \\
\hline 2321 & - & CG232I & - & & & & & & & & & & & & & & & & NP_060350.1 \\
\hline 2691 & - & CG2691 & - & & & & & & & & & & & & & & & & NP_055994.I \\
\hline 3259 & iv & CG3259 & MIP_T3 & & & $v$ & $v$ & $v$ & & $v$ & & v & & & $v$ & $v$ & $v$ & $v$ & NP_056465 \\
\hline 3344 & - & CG3344 & & Serine carboxypeptidase & & & & & & & & & & & & & & & NP_067639.1 \\
\hline 3603 & - & CG3603 & & Rhodopsin-like receptor & & & & & & & & & & & & & v & & NP_055049.1 \\
\hline 3723 & 3 & Dhc93AB & DNAH9 & ATPase activity, coupled & $v$ & $v$ & $v$ & & v & & & & & & & v & $v$ & $v$ & NP_001363 \\
\hline 3769 & 63 & CG3769 & xbxI/D2LIC & & & $v$ & $v$ & & & & $v$ & & & & & $v$ & $v$ & $v$ & NP_057092 \\
\hline 4135 & - & beat-llb & & IgSF protein, axon guidance & & & & & & & & & & & & & & & I to many \\
\hline 4314 & - & st & scarlet & ATPase & & & & & & & & & & & & & & & I to many \\
\hline 4536 & 6 & iav & TRPV4 & & & & & & & & & & & & & & $v$ & & NP_067638.3 \\
\hline 4857 & - & CG4857 & - & & & & & & & & & & & & & & & & - \\
\hline 4984 & - & CG4984 & - & & & & & & & & & & & & & & & & $N_{-} /|14| 02.2$ \\
\hline 5148 & - & CG5/48 & - & & & & & & & & & & & & & & & & - \\
\hline 5155 & $\mathrm{Neg}$ & CG5/55 & - & Binding & & & & & $v$ & & & & & & & $v$ & $v$ & $v$ & NP_060546 \\
\hline 5599 & - & CG5599 & & Acetyltransferase & & & & & & & & & & & & & $v$ & $v$ & NP_001909 \\
\hline 6054 & $\mathrm{Neg}$ & Su(fu) & Suffu $)$ & Kinase inhibitor & & & & & & & & & & & & & & & NP_057253.2 \\
\hline 6121 & $\mathrm{Neg}$ & Tip60 & & Histone acetyltransferase & & & & & & & & & & & & & $v$ & & NP_874369.1 \\
\hline 6129 & 6/iv & CG6129 & rootletin & & & & & & $v$ & & & & & $v$ & & $v$ & & $v$ & NP_055490 \\
\hline 6405 & - & CG6405 & - & & & & & & & & & & & & & & & & XP_936274.2 \\
\hline 6665 & - & CG6665 & - & & & & & & & & & & & & & & & & NP_056264.I \\
\hline 6696 & - & CG6696 & & Meprin A/proteolysis & & & & & & & & & & & & & & $v$ & NP_005916 \\
\hline 7104 & - & Spz3 & & & & & & & & & & & & & & & & & - \\
\hline 7669 & - & CG7669 & & & & & & & $v$ & & & & & & & v & & & NP_002815.3 \\
\hline 7754 & - & iotaTry & & Trypsin & & & & & & & & & & & & & & & I to many \\
\hline 8344 & - & RpllII 28 & & RNA polllllb & & & & & & & & & & & & & $v$ & & NP_060552.3 \\
\hline 8362 & $\mathrm{Neg}$ & nmdynD7 & & Nucleoside diphosphate kinase & $v$ & $v$ & $v$ & & & $v$ & & & v & $v$ & $v$ & $v$ & $v$ & $v$ & NP_037462 \\
\hline 8853 & 23 & CG8853 & IFT55/Hippi & & & $v$ & $v$ & $v$ & $v$ & $v$ & $v$ & $v$ & & & & $v$ & $v$ & $v$ & NP_060480 \\
\hline 8971 & $\cdot$ & fh & frataxin like & & & & & & & & & & & & & & & & NP_000135.2 \\
\hline 9035 & - & Tapdelta & & Signal sequence binding & & & & & & & & & & & & & & & NP_006271.I \\
\hline 9227 & iv & tectonic & Tectonic & & & & v & $v$ & & & $v$ & $v$ & & & & v & $v$ & $v$ & NP_085055 \\
\hline 9298 & - & CG9298 & & Synbindin & & & & & & & & & & & & & & & NP_057230.1 \\
\hline 9363 & - & CG9363 & & Glutathione transferase zeta & & & & & & & & & & & & & & & NP_665877.I \\
\hline 9467 & - & CG9467 & & V-gated K channel complex & & & & & & & & & & & & & & & NP_057205.2 \\
\hline 9689 & - & CG9689 & - & & & & & & & & & & & & & & & & - \\
\hline 9764 & - & yrt & yurt & Cytoskeletal protein binding & & & & & & & & & & & & & & & NP_060894.2 \\
\hline 10035 & - & CG10035 & - & & & & & & & & & & & & & & & & - \\
\hline 10068 & - & CG10068 & - & & & & & & & & & & & & & & & & NP_7|49|3.1 \\
\hline 10143 & - & Adgf E & & Adenosine deaminase & & & & & & & & & & & & & & & NP_803|24.I \\
\hline 10601 & - & mirr & & Protein binding & & & & & & & & & & & & & & $v$ & NP_0773II \\
\hline 10859 & - & CG10859 & DIC & Motor activity & & & & & & & & & & & & & & & NP_075462 \\
\hline 11015 & - & CGIIOI5 & $\mathrm{CO} 5 \mathrm{~B}$ & Cytochrome-c oxidase & & & & & & & & & & & & & $v$ & & NP_001853 \\
\hline 11164 & - & CGIII64 & - & & & & & & & & & & & & & & & & NP_078846.I \\
\hline 11356 & - & CGII356 & ARLI3B & GTP binding & & & & & & & & & & & & & & & NP_878899 \\
\hline 11426 & - & CGII426 & & Lipid metabolism & & & & & & & & & & & & & & & I to many \\
\hline 11438 & - & CGI/438 & & G protein coupled pathway & & & & & & & & & & & & & & & NP_7957/4.1 \\
\hline 11529 & - & CGII529 & & Chymotrypsin activity & & & & & & & & & & & & & & & NP_005037.I \\
\hline 11983 & - & CGII983 & - & & & & & & & & & & & & & & & & - \\
\hline 12020 & - & CG 12020 & - & Heat shock protein binding & & $v$ & & & & $v$ & & & & & & $v$ & & & NP_705842.2 \\
\hline 13125 & 5 & $C G / 3 / 25$ & & LRR & $v$ & v & $v$ & & & & & & & & $v$ & v & $v$ & $v$ & NP_II 2584 \\
\hline 13202 & - & CG13202 & - & & & & & & & & & & & & & & & & - \\
\hline 13216 & - & CGI3216 & - & & & & & & & & & & & & & & & & - \\
\hline 13251 & - & CGI325I & - & & & & & & & & & & & & & & & & NP_001012524.1 \\
\hline 13271 & - & Ugt $36 \mathrm{Bb}$ & & Glucuronosyltransferase & & & & & & & & & & & & & & & I to many \\
\hline 13415 & 2 & Cby & Chibby & & & & & & & & & & & & & & & & NP_056188.1 \\
\hline 13432 & $\mathrm{Neg}$ & I(2) 05510 & - & & & & & & & & & & & & & & & & - \\
\hline 13809 & 172/iv & osm-1 & IFTI72/wim & & & v & $v$ & $v$ & $v$ & & v & $v$ & & & & $v$ & $v$ & $v$ & NP_056477 \\
\hline 14079 & - & CGI 4079 & - & & & & & & & & & & & & & & & & NP_061946.I \\
\hline 14127 & $\mathrm{Neg}$ & $C G / 4127$ & - & & & & & & v & & & & & & & v & $v$ & $v$ & NP_659482 \\
\hline 14313 & - & $C G / 43 / 3$ & - & & & & & & & & & & & & & & & & - \\
\hline 14430 & - & CGI4430 & - & & & & & & & & & & & & & & & & - \\
\hline 14661 & - & CGI4661 & - & & & & & & & & & & & & & & & & - \\
\hline 14791 & $\mathrm{Neg}$ & Rab27 & & GTP binding & & & & $v$ & & & & & & & & $v$ & v & $v$ & NP_00457I \\
\hline 15148 & 124 & $b t v$ & $\mathrm{DNCH} 2$ & ATPase activity, coupled & & $v$ & $\mathrm{v}$ & & $v$ & & & & & & & $v$ & $v$ & $v$ & NP_001073932 \\
\hline
\end{tabular}


Table 3 (Continued)

\begin{tabular}{|c|c|c|c|c|c|c|c|c|c|c|c|c|c|c|}
\hline 15161 & 207 & CGI5161 & IFT46/dyf-6 & & $v$ & $v$ & $v$ & $v$ & $v$ & & $v$ & $v$ & $v$ & NP_064538 \\
\hline 15564 & - & CGI5564 & - & & & & & & & & & & & - \\
\hline 15878 & - & CGI5878 & - & & & & & & & & & & & - \\
\hline 17150 & 2 & CGI7150 & DNAH3 & Motor activity & $\mathrm{v}$ & $\mathrm{v}$ & & & & & $v$ & & & NP_060009 \\
\hline 17259 & - & CGI7259 & & Seryl trna synthetase & & & & & & & & $v$ & & NP_006504.2 \\
\hline 17284 & - & Obp93a & & Odorant binding & & & & & & & & & & - \\
\hline 17785 & $\mathrm{Neg}$ & Golgin84 & & & & & & & & & & & & NP_005104.2 \\
\hline 18432 & - & CGI8432 & & & & & & & & & & & & NP_001073991.1 \\
\hline 18584 & - & CGI8584 & UNC84 & & & & & & & & & & & NP_056189.1 \\
\hline 18869 & - & CGI8869 & & UDP glycosyl transferase & & & & & & & & & & - \\
\hline 30021 & - & skf & & Guanylate kinase & & & & & & & & & & NP_775767.2 \\
\hline 30441 & 2.8 & CG3044I & IFT20 & & $\mathrm{v}$ & & $v$ & & $v$ & $v$ & $v$ & v & & NP_777547.I \\
\hline 31036 & 4/iv & CG31036 & - & & & & & & & & & & & - \\
\hline 31127 & - & Wsck & & Protein-tyrosine kinase & & & & & & & & & & NP_005223.3 \\
\hline 31257 & - & CG3/257 & - & & & & & & & & & & & - \\
\hline 31321 & - & CG3/321 & - & & & & & & & & & & & NP_542400.2 \\
\hline 31640 & - & CG31640 & - & Protein-tyrosine kinase & & & & & & & & & & NP_001014796.1 \\
\hline 31824 & - & CG3/824 & - & Trypsin & & & & & & & & & & - \\
\hline 33038 & $\mathrm{Neg}$ & CG8433 & exostosin2 & & & & & & & & & & & NP_056499.2 \\
\hline
\end{tabular}

Listing established with the restricted GYTRYYN\{I-3\}RRHRAC X-box consensus. Neg, invariant expression in $d R f x$ deficient background compared to $\mathrm{wt}$; iv, in vivo confirmation of reporter construct down regulation in $d R f x$ deficient background compared to wild type. The presence of a gene in other published studies is noted as $v$.

genome wide $\mathrm{X}$-box consensus motif search allowed the establishment of promising sets of candidate genes for ciliogenesis studies.

\section{Functional analysis of identified $\mathbf{X}$-box genes}

We performed functional expression studies to determine whether or not some of the 83 X-box genes (Table 3) are indeed under dRFX control and if they are involved in ciliogenesis. Twenty-five genes were tested by real time RT-PCR to compare their levels of expression in wild-type versus $d R f x$ deficient fly samples. Interestingly, 16 are under dRFX control (Table 3, fold variation indicated in column 2). Among them, 11 have not yet been described as RFX targets in any biological system and two of them have no assigned function as of yet. Nine genes were not found to be under dRFX control (Table 3, noted as 'Neg' in column 2). Among 19 genes also represented in the DCBB dataset (Table 3, Additional data file 2), 17 were tested by real time PCR. Fourteen are indeed regulated by dRFX and only three do not appear to be regulated by it. The two remaining genes were not amplified by real time RT-PCR and, thus, could not be analyzed by this approach. Interestingly, among six genes that were not found in any ciliary database and whose expression was quantified by real-time PCR, two (CG13415/Cby, CG31036) were downregulated in $d R f x$ mutants. Thus, a high proportion of the genes on the list of $83 \mathrm{X}$-box genes are indeed dRFX target genes. The 58 remaining genes from this list that have not yet been analyzed are thus promising candidates. Our whole genome screen led to the identification of novel dRFX target genes.

Among the 11 novel dRFX target genes that we identified in this screen and that have never been described as RFX target genes in any organism, 9 do have a described or highly predic- tive function in ciliogenesis in other organisms. For example, CG15161 encodes the homolog of the IFT46 subunit in Chlamydomonas [50] and the dyf-6 ciliary gene in C. elegans [51]. CG15148/btv, CG3723 and CG1715O encode different dynein subunits. beethoven (btv) mutants show defects in sensory cilia in Drosophila [52], whereas no functional studies are available for either $C G 3723$ and $C G 17150$ or their orthologs in any biological system. CG6129 is the only Drosophila member of the rootletin family of proteins. In mammals, rootletin is necessary for retinal cilia stability and centrosome cohesion in mammalian cells [53-56]. CG4536/ osm-9 encodes a vanilloid receptor of the transient receptor potential (TRP) family of ion channels. osm-9 is involved in sensory cilia function in Drosophila and C. elegans, and in mammals, TRPV4 plays a crucial role in ciliary activity [57]. CG9227/Tectonic has been described as being involved in Shh signaling in mouse [58]. It has been isolated by comparative genomics as a candidate for ciliogenesis and shown to be specific to ciliated cells in Drosophila [13]. CG13125 has recently been shown to be specific to species with motile cilia and its homolog, $\mathrm{TbCMF} 46$, is necessary for flagellar motility in T. brucei [59]. CG3259 encodes the MIP-T3 protein that associates with the tumor necrosis factor receptor in human cells. It is also an inhibitor of the IL13 signaling pathway that is known to repress ciliary differentiation of human epithelial cells in vitro [60-62]. It is expressed in ciliated sensory cells in Drosophila [13]. Thus, the gene CG3259 may have a direct function in ciliogenesis, which functional studies in Drosophila will allow to be deciphered.

Interestingly, two novel dRFX target genes have not been described as being involved in ciliogenesis in any organism. CG13415/Chibby encodes a protein that interacts with the $\beta$ catenin protein and has been shown in Drosophila and in 
Comparisons of Drosophila X-box candidate genes with the Drosophila cilia and basal body genes

\begin{tabular}{|c|c|c|c|c|}
\hline & \multirow[t]{2}{*}{ Total no. of genes } & \multicolumn{3}{|c|}{ X-box gene candidates } \\
\hline & & Total & Conserved X-box & Restricted consensus \\
\hline In D. melanogaster & 14,500 & 4,726 & & \\
\hline In D. pseudoobscura & 10,000 & 3,848 & & \\
\hline No. of homologous genes in both species & 9,815 & 1,462 & 412 & 83 \\
\hline No. of DCBB genes in D. melanogaster & 815 & 129 (15.8\%*) & 47 & 19 \\
\hline Stolc et al. [15] & 88 & $28(31.9 \% \dagger)$ & 14 & 6 \\
\hline Ostrowski et al. [10] & 126 & $28(22.2 \% \dagger)$ & 13 & 9 \\
\hline Pazour et al. [II] & 192 & $4 \mathrm{I}(21.3 \% \dagger)$ & 18 & 12 \\
\hline Avidor-Reiss et al. [13] & 188 & $38(20.3 \% \dagger)$ & 14 & 8 \\
\hline Li et al. $[14]$ & 260 & $50(19.2 \% \dagger)$ & 25 & 12 \\
\hline Blacque et al. [37] & 50 & $9(18.0 \% \dagger)$ & 6 & 5 \\
\hline Broadhead et al. [12] & 69 & $15(22.1 \% \dagger)$ & 5 & 3 \\
\hline Efimenko et al. [36] & 117 & $20(16.7 \% \dagger)$ & 8 & 6 \\
\hline Keller et al. [45] & 51 & $9(17.7 \% \dagger)$ & 3 & I \\
\hline Andersen et al. [44] & 56 & $8(14.3 \% \dagger)$ & 2 & 2 \\
\hline Percent $X$-box genes in the DCBB dataset & & $\mathbf{8 . 8 2} \%$ & $11.41 \%$ & $22.89 \%$ \\
\hline
\end{tabular}

* Percent of the 815 different DCBB genes. Percent of total genes for each study present in the X-box list. See Additional data file 2 for the DCBB dataset.

mammalian cells to antagonize the $\mathrm{Wg} / \mathrm{Wnt}$ signaling pathway [63-65]. The second gene, CG31036, has an unknown function and no obvious ortholog in vertebrates. Protein structure prediction algorithms detect a central transmembrane domain and a signal peptide at the amino-terminus of the protein encoded by $C G 31036$.

\section{Expression profile of three novel dRFX target genes}

In order to further validate our screen, we chose three genes (CG6129/rootletin, C13125/TbCMF46 and CG31036) for in vivo study. CG6129 was selected to address the question of conservation in Drosophila of the dual role described in mammals for the rootletin protein in centrosome and ciliary biology. CG13125 is of particular interest to evaluate the possible involvement of a 'motility gene' in Drosophila sensory cilia. Last, since nothing was known about $C G 31036$, we wanted to address whether this gene is involved in ciliogenesis and, thus, validate the overall X-box screening strategy.

Reporter constructs were made by cloning large promoter fragments including the conserved X-box, plus coding sequences in frame with green fluorescent protein (GFP). Transgenic flies were established and analyzed for GFP expression. Two types of ciliated cells have been described in Drosophila: spermatozoa and type I sensory neurons that innervate the proprioceptive chordotonal organs and external sensory organs that are mechano- or chemosensory. Remarkably, the expression of all three reporter constructs was observed only in type I sensory neurons. As a control, reporter GFP expression was compared to mRNA expression by in situ hybridization. CG6129/rootletin protein expression reproduces the expression of the transcript in only type I sensory neurons of the embryo (data not shown). CG31036 RNA expression is also available from the BDGP database [66]. CG31036 mRNA is restricted to type I sensory neurons of the head, thoraxes and abdomen of the embryo and reflects the protein expression of our transgene. However, we did not observe a strong protein expression in the gut as observed for the transcript. This could either reflect a non-specific hybridization signal or the presence of other transcript isoforms driven by a different promoter. We could not detect CG13125 transcripts by in situ hybridization, likely illustrating the faint expression of this gene in Drosophila.

Chimeric CG6129::GFP protein was present in the rootlet processes of the chordotonal dendrites, in agreement with the predicted function of rootletin in ciliary rootlet organization (Figure 4). It was also detected faintly at the cilium tip (Figure 4d) and clearly in axons (Figure 4). Since our construct does not include all the coding sequences of the rootletin protein, it is possible that the GFP expression does not reflect the exact location of the endogenous protein. Rootletin has been shown in mammalian cell culture to be localized to the ciliary rootlet and to be involved in centrosome cohesion [56]. We show that CG6129/Rootletin expression is restricted to ciliated chordotonal neurons in Drosophila, thus suggesting an involvement only in ciliogenesis. Despite strong GFP expression in the chordotonal organs, no expression was observed in the ciliated sensory neurons that innervate external sensory organs. Either the expression in those cells is too weak, or ciliary rootlets in Drosophila, as represented by CG6129/rootletin GFP 


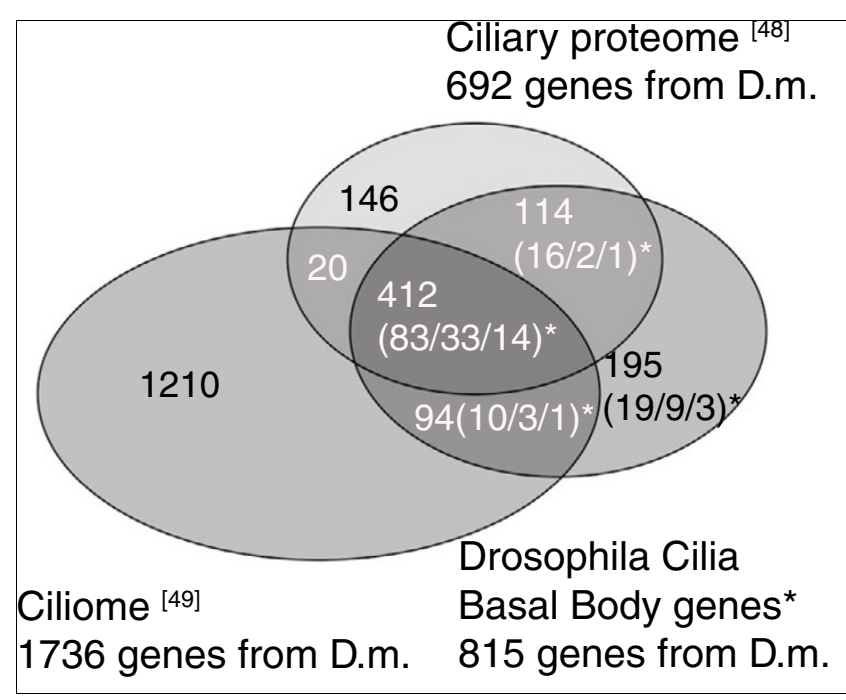

Figure 3

Comparison of the DCBB set of genes with the Ciliary proteome and Ciliome databases. Venn diagram presenting the overlaps between the three datasets: the cilia proteome [46,48]; the ciliome [47,49], and the DCBB (Additional data file 2). Asterisks indicate this study. Note that only 412 common genes are found in the three datasets. The number of genes also found in the I,462, 4I 2 or 83 X-box gene lists (Table 4), respectively, are noted in parentheses. The numbers of genes selected in the different studies to construct each dataset are given in Additional data file 3.

expression, are restricted only to chordotonal organs, as observed previously by electron microscopy $[67,68]$.

CG31036::GFP specifically marks the ciliated endings of chordotonal neurons and confirms that this novel protein is a component of ciliated endings (Figure 4). The GFP signal is apposed to the $21 \mathrm{~A} 6$ antibody staining, directed against the eyes shut protein, which has been described to locate at the ciliary dilation around the tip of the ciliated segment [69]. This implies that CG31036::GFP most likely locates to the tip of the tubular bundle that extends after the ciliary dilation (schematic in Figure 1a). However, only ultrastructural observations of immunogold labelings will allow precise subcellular localization of both CG6129/rootletin and CG31036. Interestingly, CG31036::GFP expression is also detectable in external sensory neurons as a dot apposed to the 21A6 antibody staining (Figure 4f). Finally, we confirmed that both reporter constructs are under $d R f x$ control as the GFP signal was completely shut down in a $d R f x$ mutant background (compare Figure 4d and 4e or $4 \mathrm{i}$ and $4 \mathrm{j}$ ).

For the third construct, CG13125::GFP localization was consistently observed in the chordotonal neurons at the base of the cilium, presumably the basal body region, and also at the tip of what is likely the cilium. GFP expression was also often observed in the external sensory neurons as a dot but without consistent reproducibility, probably illustrating a threshold level of expression for these cells and the faint level of expression of the CG13125/TbCMF46 transgene (Figure 4k,l).
In conclusion, the three novel dRFX target genes that we identified in our X-box motif searches are indeed under dRFX control in vivo and specifically expressed in ciliated sensory neurons in Drosophila. In addition, they encode proteins that are localized to the base or the tip of the cilium, thus suggesting a role in ciliary structure or function.

\section{Discussion}

Ciliogenic RFX regulatory networks are conserved between $C$. elegans and D. melanogaster. Based on these first observations, the genomic screens we conducted combined with functional and in vivo gene analyses led to the identification of at least 11 novel genes that had never been described as RFX targets in any biological model. In addition, our screen allowed us to identify at least two novel genes specifically expressed in ciliated sensory neurons in Drosophila that are potentially involved in sensory ciliogenesis. These results validate the accuracy of our screens. Our work thus provides a new set of candidate genes for further functional studies in ciliogenesis.

\section{Molecular nature of RFX target gene products}

Our Drosophila genome wide X-box screen led to the identification of $83 \mathrm{X}$-box genes among which we report 11 novel RFX targets. Combined with the genes identified by comparisons to C. elegans or to other genomic studies in Drosophila (Table 1) [13], we report 35 genes regulated by dRFX in Drosophila. Most of these genes can be classified based on their described function. Many of the RFX target genes are involved in IFT, which is necessary for cilium assembly and function [1]. Remarkably, a second class of genes regulated by dRFX includes all the Drosophila homologs of $B B S$ genes. Similarly, most $C$. elegans $B B S$ genes are regulated by DAF19 [14,36,37]. This strong dependence of $B B S$ genes on RFX control may thus be conserved in mammals. Hence, RFX proteins may be involved in BBS in humans. Interestingly, two of the three Drosophila genes coding for proteins with B9 domains are also controlled by dRFX (tectonic, CG14870). One human B9 domain protein, MKS1, is known to be involved in the human Meckel-Gruber syndrome [70]. The molecular function of this domain is unknown and work in Drosophila suggested that these two B9 domain containing proteins are likely involved in ciliogenesis [13]. Several of the novel dRFX target genes that we identified in this study encode known components of the ciliary axoneme and associated structures, such as axonemal dyneins or rootletin. Other genes encode different types of proteins likely involved in sensory transduction (CG4536/osm-9/TRPV4 or MIP-T3). A last class includes genes for which the function is either not described or poorly understood, such as CG31036 and CG13125. However, our functional studies strongly suggest that they are also probably involved in sensory ciliogenesis in Drosophila as well. Thus, RFX target genes play various roles in ciliary structure and function and our X-box search strategy has proven to be useful to identify novel ciliogenic genes. 


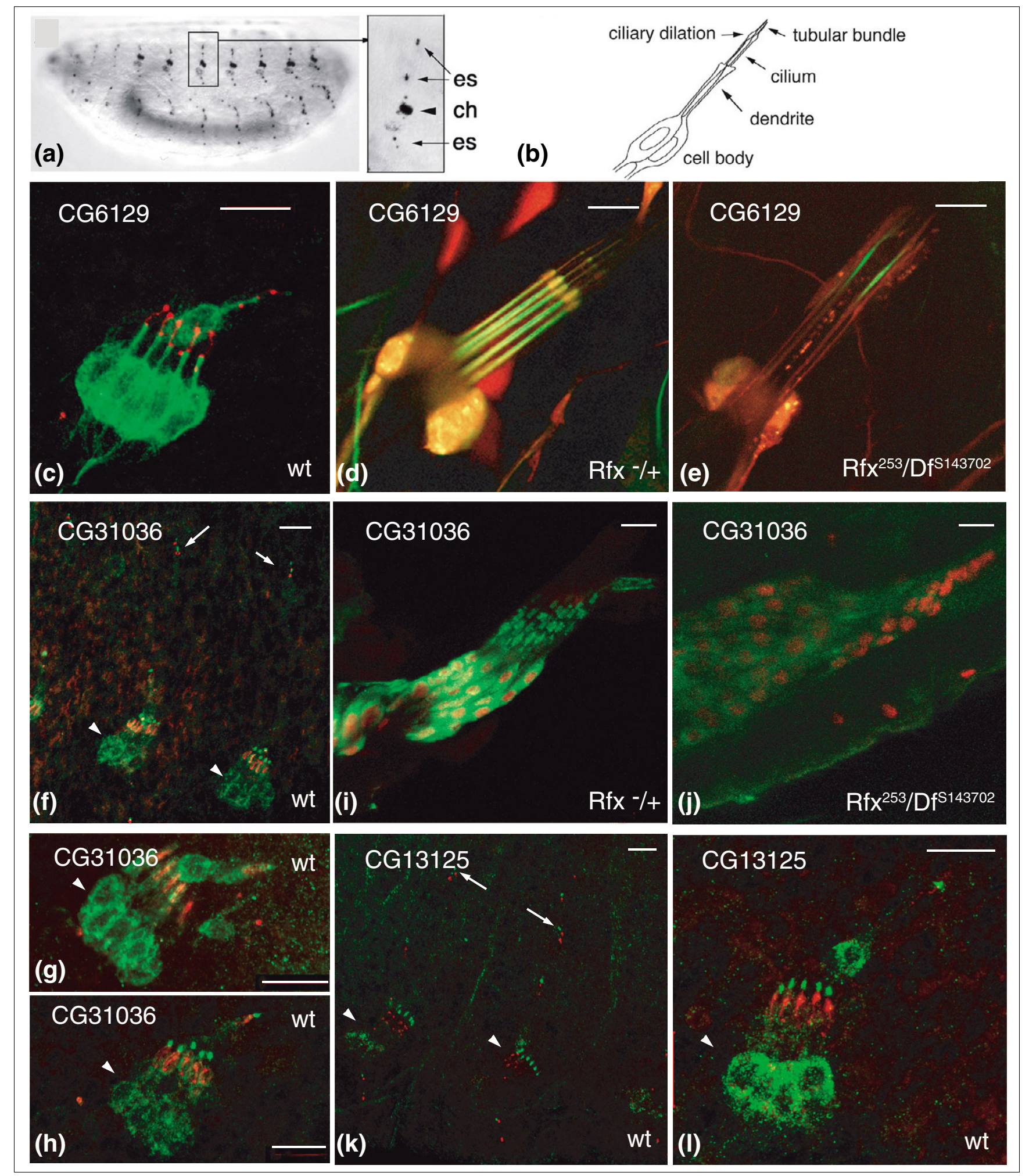

Figure 4 (see legend on next page) 
Figure 4 (see previous page)

Reporter GFP expression studies for three X-box containing genes. (a) Stereotypical arrangement of type I sensory neurons in a Drosophila embryo, anterior to the left, stained with the 2 IA6 antibody with a magnification of the dorsal and lateral neurons of one abdominal segment as visualized in (f,k). The arrowhead indicates the five lateral chordotonal neurons (ch) and the arrows point to the neurons of the external sensory (es) organs. (b) Schematic of two typical chordotonal organs of Drosophila. (c-I) Confocal imaging of GFP expression of transgenic lines carrying the promoter region and coding sequences fused to the GFP for CG6/29/rootletin (c-e), CG3 I036 (f-h) and CGI3/25/TbCMF46 (k,I). GFP expression is only observed in ciliated sensory neurons of $D$. melanogaster where the chimeric GFP proteins are localized to the ciliary apparatus. (c) CG6/29::GFP reporter expression (green) is observed in embryonic chordotonal organs, mainly along the dendrite from the base of the cilium to the cell body. The 2 IA6 antibody (red, see Materials and methods) labels the ciliary dilation of the cilium. (d,e) Live GFP imaging of the lateral pentascolopidial chordotonal organs in male third instar larvae of $d R f x$ deficient (e) and control (d) sibs. The elav-RFP expression (red) labels all neurons. CG6/29/rootletin is regulated by dRfx as no GFP expression is observed in $d R f x$ deficient larvae. (f-h) CG3 1036::GFP reporter expression (green) is observed both in the external sensory neurons (arrows in (f)) and the chordotonal neurons in the embryo (arrowhead in (f-h)). The 21 A6 antibody (red) labels the ciliary dilation at the tip of the dendrite. CG3 I036::GFP protein localization appears to be slightly different depending on the fixative used (paraformaldehyde in $(\mathrm{g})$, methanol in $(\mathrm{h})$ ). ( $\mathrm{i}-\mathrm{j})$ Immunodetection of CG3 1036::GFP expression in leg chordotonal organs of 72-hour pupae in dRfx deficient (j) or control (i) sibs. The anti-ELAV antibody (red) labels all neurons. No CG3 1036::GFP expression is observed in $d R f x$ deficient pupae (j). (k,l) CGI3/25::GFP expression is observed by immunodetection in the embryonic chordotonal organs (arrowheads in $(k, l))$ but also in the external sensory neurons (arrows in $(k)$ ). A higher magnification of the lateral chordotonal organs (I) shows that GFP is apposed to the $21 \mathrm{~A} 6$ immunostaining (red). Scale bar $=10 \mu \mathrm{m}$.

\section{Database mining using the $X$-box promoter motif}

This full set of dRFX target genes in Drosophila is of crucial importance, as we can now more precisely define X-box sequences and the promoter context required for dRFX control. This will be particularly useful for further database mining of dRFX target genes in Drosophila. In fact, several genes that are under dRFX control (Table 1, for example CG4525, CG17599) for which an X-box can be identified did not come out in the whole genome X-box screen. Several reasons can explain this result. First, homologs were not all annotated in CDS listings that were available at the time of the search (for example, CG18631, CG9595, nompB in D. pseudoobsura). Second, annotation of both Drosophila databases is incomplete, as sometimes the start codon is not properly defined for all genes. Our X-box search algorithm keeps only genes for which the X-box match is upstream of the ATG. For example, for $C G 15666 / G A 13881$, we clearly predict that the correct ATG should be considered 75 bp downstream of the currently defined ATG, based on evolutionarily conserved sequences. This definition clearly excludes the homologous genes $C G 15666$ and $G A 13881$ from the dataset. However, as illustrated in Table 2, in a few cases, our X-box consensus cannot define a clearly conserved X-box match in the two Drosophila species for genes that appear to be down-regulated in a $d R f x$ mutant, while several individual X-boxes are found separately in each organism. This could either reflect that these genes are not direct dRFX targets but are shut down by a feedback control loop that is not dependent on a Xbox motif, or that the X-box is only loosely conserved in some promoter contexts. Notably, homologs of these genes in $C$. elegans are under RFX (DAF-19) control and have a well defined X-box (for example, CG9333/che-2, CG13691/bbs8 ), which argues in favor of the second possibility. Interestingly, we also quantified the expression levels in control and dRfx deficient Drosophila of several genes of the DCBB dataset that did not come out of the X-box genome-wide motif search. It allowed us to identify several novel genes that are indeed down-regulated in $d R f x$ mutants, but for which no conserved X-box can be recognized based on our initial con- sensus motif (AL, unpublished). Altogether, our observations clearly highlight the difficulties encountered in motif definition in promoters. Similar conclusions were deduced from a parallel approach performed in C. elegans, which has led to the identification of several novel DAF-19 target genes [38]. Interestingly, in that study the in silico search was associated with microarray analysis of transcripts in wild-type and daf19 mutant worms. The in silico search allowed the identification of $93 \mathrm{X}$-box genes. Yet, among the 466 genes that were shown to be down-regulated at least two-fold in microarray hybridization experiments, only 25 were also represented in the 93 in silico X-box gene list. Thus, in silico searches on isolated motifs are likely hampered by a high level of false negatives. In order to improve the screening efficiency, the use of combinatorial motif searches would probably greatly enhance the accuracy of the screen as proposed by other studies $[71,72]$. Even though, since conserved X-boxes that we identified are rarely associated with highly conserved surrounding sequences (Table 2), it is reasonable to assume that other conserved nearby motifs, still to be identified, could help to discriminate between false positives and false negatives.

\section{Regulatory network of ciliary genes}

We have identified 35 genes that are transcriptionally downregulated in $d R f x$ mutants. We show that RFX regulatory networks are conserved between C. elegans and Drosophila as most of the genes controlled by DAF-19 in C. elegans are also under dRFX control in D. melanogaster. Interestingly, our results show that only certain subsets of ciliogenic genes are regulated by RFX proteins. For example, in our assay conditions all the genes known to be involved in IFT-A complexes are not regulated by dRFX, whereas all IFT-B homologous proteins are regulated by dRFX. In addition, retrograde motors are also regulated by $\mathrm{dRFX}$ (CG15148/btv and CG3769), whereas anterograde motors seem not to be. Indeed, in addition to $C G 10642 / K_{I F} A$, the main described anterograde motor in several organisms, we have shown that two other kinesin subunits, CG17461/Kif3C/osm-3 and $C G 7293 / K l p 68 D$, are invariantly expressed in wild-type and 
$d R f x$-deficient Drosophila (AL, data not shown). It is also interesting to note that all the $B B S$ gene homologs in $D$. melanogaster are under dRFX control (Table 1).

The biological significance of these observations is unclear. It could reflect the fact that IFT-B proteins, BBS proteins and the dyneins involved in IFT are dedicated to ciliogenesis and, therefore, need to be turned on concomitantly only when the cilium is formed, whereas IFT-A complexes or anterograde transport kinesin II share more complex regulatory controls as they might be necessary also for other cellular functions. This is the case for kinesin II motors [73], but does not seem to be true for IFT-A complexes as these proteins are proposed to be specific for ciliated organisms [13]. In C. elegans, the ciliary IFT machinery works in modular fashion [74], and it is tempting to speculate that RFX-dependent proteins could be involved in specialized ciliogenic transport modules.

Genes necessary for centriole biogenesis or replication, such as the recently described DSas-6, DSas-4 or sak genes [75-78] are not present in our screen and no conserved X-box can be found upstream of these genes. Thus, dRFX does not seem to regulate centriole biogenesis and appears to be restricted to cilia assembly only.

To find which transcription factors are responsible for governing other sets of ciliary proteins will certainly be one track to follow. Based on our data, it would be of particular interest to compare promoter sequences of genes, either regulated by $\mathrm{dRFX}$, or not. It may allow us to discover novel regulatory motifs and protein modules that are necessary to coordinate ciliogenesis control. So far, only a few transcription factors have been shown to be involved in the control of ciliogenesis: the RFX proteins [21,23,24], Foxj1 [16], and HNF1-beta [17]. However, the last two have no obvious homologs in Drosophila. Thus, our work strongly suggests that novel transcription factors necessary for ciliogenesis still need to be discovered.

\section{Novel RFX target genes}

Some of the novel RFX target genes found in Drosophila were unexpected. For example, we identified several proteins that are proposed to be involved in flagella or cilia motility, such as dynein heavy chains (CG1715O/Dhc93AB). Recently, a $C G 13125$ homolog has also been shown to function as a motility factor in T. brucei (TbCMF46) [59]. Sensory cilia are thought not to be motile in general. However, it has been shown that Drosophila chordotonal neurons of the antenna generate motion that depends on the integrity of proteins encoded by genes such as CG15148/btv (cytoplasmic dynein heavy chain) or CG1462O/tilB (LRRC6 homolog), described to affect the axonemal structure [52,79] (D Eberl, personal communication). In addition, cilia of the chordotonal neurons of the grasshopper bend upon vibration stimulation [80]. Thus, proteins involved in axonemal motility might be important for motion generation of the cilium in response to mechanical stimulation. It will be of high interest to determine whether flies defective in these 'motility' genes are affected in hearing and, more specifically, in the motility of the mechanosensory cilium that amplifies hearing vibrations. Interestingly, $C G 13125 / T b C M F 46$ does not seem to be expressed in fly testis (AL, unpublished), where the spermatozoa are the only cell type with a motile flagellum in flies. This suggests that like CG15148/btv, CG13125/TbCMF46 function could be restricted to the sensory cilium and, more specifically, in allowing these cilia to mechanically respond to auditory vibrations [52]. Thus, our data suggest that in the fly, possible axonemal motility could be regulated by different subsets of proteins in sperm flagella and in mechanosensory cilia. This is of particular interest with regard to hearing in mammals, which is dependent on hair cell motility. It will be very interesting to determine whether the CG13125/ TbCMF46 homolog in mammals does have a specific function in those cell types.

We also identified in our screen three genes (CG6054/Su(fu), $C G 13415 / C b y, C G 33038 / E x t(2))$ known to be involved in the hedgehog or wingless signaling pathways in Drosophila. $S u(f u)$ and $\operatorname{Ext}(2)$ are involved in the Hedgehog pathway and $\mathrm{Su}(\mathrm{fu})$ is localized to cilia in mammalian cells [81]. However, $S u(f u)$ and $\operatorname{Ext}(2)$ do not appear to be under $d R f x$ control according to real-time PCR quantification results (Table 3) and may be false positives in our screen. This result argues in favor of the generally accepted observation that the Hedgehog signaling pathway does not seem to depend on ciliogenic proteins in Drosophila [82]. Only Chibby (Cby) is statistically down-regulated two-fold in a $d R f x$ deficient background. $C b y$ was isolated in a two-hybrid screen for armadillo/beta-catenin interactors. RNAi knock-down of Cby in Drosophila embryos leads to ectopic activation of the wingless pathway [63]. Cby is also described to antagonize the Wnt/beta-catenin pathway in mammalian cells $[64,65]$. However, the expression pattern of Cby in Drosophila is not documented, so we do not know if the variations of expression observed in the $d R f x$ deficient background are connected to $d R f x$ expression and, thus, if it is biologically significant.

Among the 83 genes with conserved X-boxes between $D$. melanogaster and D. pseudoobscura (Table 3), several genes were hardly detectable by quantitative RT-PCR. Hence we were unable to determine by this approach if they are under dRFX control. This could reflect that these genes are expressed only in a subset of sensory neurons and, thus, difficult to detect by quantitative RT-PCR. Nevertheless, several genes are interesting as potential ciliogenic or RFX target genes. For example, CG14079 is homologous to a mouse protein that appears to be specific to testis. CG11356 is homologous to mammalian arl13, which has just been isolated in an ethyl-nitroso-urea screen for neural tube defects in mouse. Indeed, mutation of arl13 affects ciliary architecture and Sonic-Hedgehog signaling in mouse [83]. This gene, $C G 11356$, was not found in any previous ciliogenesis study, 
again illustrating the accuracy of our screen. Functional studies in Drosophila will be of particular importance to demonstrate the role of this gene in sensory ciliogenesis.

\section{Conclusion}

We have identified more than 30 dRFX target genes in Drosophila by exploiting the efficiency of the X-box promoter motif search by using two divergent Drosophila species in a comparative approach. These full sets of RFX dependent or independent ciliary genes are of particular importance for studies of X-box promoter motifs and associated promoter contexts in Drosophila. More remarkably, our screen allowed the identification of at least two novel genes specific to sensory ciliary architecture in D. melanogaster and provides several new RFX target gene candidates potentially involved in ciliogenesis. This is of particular importance with regard to the growing number of human diseases that are being associated with ciliary defects (for reviews, see $[4,5,7]$ ).

\section{Materials and methods Quantitative RT-PCR}

Total RNA was extracted from 40-hour old puparium using TRIzol reagent (Invitrogen, Carlsbad, CA, USA) or RNeazy (Qiagen, Venlo, The Netherlands). Pupae head and abdomen were removed as well as internal organs and muscles in order to enrich as much as possible the extract for sensory organs from thoraxes, legs and wings. DNA was digested with DNAfree reagent (Ambion, Austin, TX, USA). Reverse transcription (RT) was performed on $2 \mu \mathrm{g}$ of RNA derived from pools of 5 thoraxes with random hexamers (Promega, Madison, WI, USA) with RevertAid ${ }^{\text {TM }} \mathrm{H}$ Minus M-MuLV reverse transcriptase (Fermentas, Burlington, Canada). Real-time PCR analysis was performed with SYBR Green fluorescent PCR (Qiagen) in a LightCycler (Roche, Basel, Switzerland) or a MX30oo (Stratagene, Cedar Creek, TX, USA) fluorescent temperature cycler. Primer sequences specific for each gene are available upon request. Primers were used at $0.5 \mu \mathrm{M}$. PCR conditions were as follows: $95^{\circ} \mathrm{C}, 15$ minutes; $35 \times\left(95^{\circ} \mathrm{C}, 15\right.$ $\left.\mathrm{s} ; 60^{\circ} \mathrm{C}, 20 \mathrm{~s} ; 72^{\circ} \mathrm{C}, 20 \mathrm{~s}\right)$. According to melting point analysis, only one PCR product was amplified under these conditions. RNA extracted from wild-type samples was used to generate a standard quantification curve for each gene, allowing the calculation of relative amounts of transcripts in mutant samples compared to wild type. All reactions were performed with four biological replicates and two technical replicates. Results were normalized with respect to CG9874/TBP expression and standard errors of the mean were calculated. Results are expressed as relative mutant to wild-type expression ratios. Significance levels were tested with unpaired $t$-test.

\section{Bioinformatics}

Individual X-boxes (consensus RYYNYYN\{1-3\}RRNRAC) were searched for in the 5 ' upstream regions of ATGs on the same strand (+) and the antiparallel strand (-) in both D. mel- anogaster and D. pseudoobscura homologs [84]. Genome wide searches for X-box promoter motifs were primarily performed using a Perl-based algorithm that identifies all possible matches in a given DNA sequence. First, the algorithm finds all sequences that match a defined consensus, then the main module implements a cross-match file that compares a $3 \mathrm{~kb}$ window downstream of each match to a file containing the DNA sequences for all predicted genes [36]. Genome sequence information, gene prediction and CDS files for $\mathrm{X}$ box searches were obtained from the following sources: the $D$. melanogaster complete genome sequence used was BDGP release 4; the complete CDS list was built from release 3.2.1 [85]. For D. pseudoobscura the 28 August 2003 genome assembly was used and release 2.1 of CDS sequences from BCM-HGSC were used [40]. Reverse BLASTP analysis was performed between the two CDS files in order to establish a list of orthologous genes between the two fly species with a cut-off value of BLAST e-score $<1 \mathrm{e}^{-10}$. Comparisons of all listed gene information were performed on a Unix platform. BDGP and Flybase databases were mined for expression patterns and gene information. Genome conservation between the two fly species was evaluated using the VISTA interface [86].

\section{DCBB dataset}

The ciliary and basal body genes in Additional data file 2 were identified using a reverse BLASTP strategy to define the best homologous proteins or genes described in the following studies: 210 proteins published in Table 2 from the human ciliary proteome [10] as modified by Marshall [87], 159 putative target genes of DAF-19 [36], 219 over expressed genes after deflagellation in $C$. reinhardtii described in Table 9 of Stolc et al. [15], 54 genes (Table 1) expressed in ciliated sensory neurons in C. elegans [37], 654 proteins identified in $C$. reinhardtii flagella [11], 380 proteins identified in the $T$. brucei flagella proteome [12] and 114 proteins listed in Table S1 for the human cell centrosome [44]. The following Drosophila homologs were extracted from published work: 260 genes described as homologous to the FABB proteins from $C$. reinhardtii in Table $\mathrm{S} 1$ of Li et al. [14], 51 genes described as homologous to 195 proteins described in Table S2 for the basal body proteome of C. reinhardtii [45] and 187 genes from Table $\mathrm{S} 1$ of compartmentalized cilia predicted genes, which has been modified to 188 genes according to Flybase annotation [13].

\section{Reporter constructs}

DNA fragments were amplified from wild-type fly genomic DNA using the Expand Long Template PCR system (Roche). Cloning strategies used primers to clone in frame the gene of interest to the GFP sequence of the PW8-GFP vector [88]. CG13125::GFP plasmid, a 3,547 bp genomic DNA fragment containing the complete coding sequence of CG13125-RA and $\mathrm{RB}$, was amplified from Canton-S using primers starting $1,484 \mathrm{bp}$ upstream of the RB ATG until the penultimate codon of the gene. CG6129::GFP plasmid, a 4,129 bp genomic DNA 
fragment containing part of the CG6129-RB gene, was amplified by PCR from Charolles genomic DNA using primers starting 2,619 bp upstream of the RB ATG. CG31036::GFP plasmid, a 3,780 bp genomic DNA fragment containing part of the CG31036-RA gene, was amplified by PCR from Canton$\mathrm{S}$ using primers starting $1,800 \mathrm{bp}$ upstream of the ATG. All coding regions cloned were entirely sequenced prior to transgenesis. Transgenic lines were established by P-element mediated germline transformation as described [89].

The following fly stocks were used for experiments: $\mathrm{P}\left\{\right.$ mecCP:Gal437a1, $\quad \mathrm{P}\{\mathrm{Osm}-1 \text { :Gal4\} }\}^{\mathrm{T} 17 \# 7 \mathrm{a} 1}$, P\{CG9227:Gal4\} $\}^{\mathrm{T} 32 \# 10 a 2}$ and $\mathrm{P}\left\{\mathrm{CG}_{32} \text { 259:Gal4\} }\right\}^{\mathrm{T} 39 \# 13 \mathrm{a} 1}$ were gifts from Tomer Avidor-Reiss (Harvard Medical School, Boston, MA, USA). P\{UAS-RFP $\}^{31}$ was a gift from Maurice Kernan (Stony Brook University, New-York, NY, USA) and P $\{\text { GawB:elav }\}^{C 155}$ and P $\{\text { UAS-mCD8::GFP.L }\}^{L L 6}$ strains were provided by the Drosophila Bloomington Stock Center, IN, USA.

\section{Fly genetics and observations}

Fly genotypes used to extract RNA were st $d R f x^{253 e} \mathrm{ca} /$ $D f^{S 143702}$ for mutants and st e ca/D $f^{S 143702}$ for control flies [23]. Control and mutant flies presented in Figure 1 share the same genotype with the exception of the third chromosome, which is heterozygous for the $d R f x^{253}$ carrying chromosome in the controls. Genotypes for flies were: oseg1 flies, $y w$ $P\{U A S: C D 8: G F P\} / Y$; $\quad P\{$ mecCP:Gal437a1 $\} /+$; $P\{U A S: C D 8: G F P\} \quad R f x^{253 / R f x} x^{49}$; osm-1 flies, $w ; P\{O s m-$ 1:Gal4 $\left.{ }^{T 17 \# 7 a 1}\right\} /+; \quad P\{U A S: C D 8: G F P\} \quad R f x^{253} / R f x^{49} ; C_{3259}$ flies, $y \quad w \quad P\{U A S: C D 8: G F P\} ; \quad$ st $R f x^{253 e}$ P\{CG3259:Gal4 ${ }^{\text {T39\#13a1 }\} / R f x 49 ;}$ and CG9227 flies, $y w$

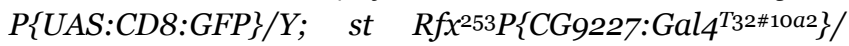
$R f x^{49}$. Control and mutant flies presented in Figure 4 were sibs sorted from the same crosses. For CG6129 expression in a $d R f x$ deficient background, females ( $w$ elav ${ }^{c 155 P}\{U A S$ $\left.\left.R F P^{31}\right\} ; \quad R f x^{49} / T M 6 B, T b\right)$ were crossed with males (w;P\{CG6129:EGFP\}M33; Rf $\left.x^{253} / T M 6 B, T b\right)$. For CG31036, the crosses were: females $(w ; P\{C G 31036: E G F P\} F 27$; $\left.D f^{S 143702} / T M 6 B, T b\right)$ with males ( $w ; P\{C G 31036: E G F P\}^{27}$; $R f x^{253 / T M 6 B, ~ T b) . ~}$

The preparation of embryos for staining assays was carried out according to general methods described previously [90]. Live observations of dechorionated embryos and larvae were performed on mounted material under coverslips in DakoCytomation media. For pupae immunostaining, 72- to 96-hour old animals were fixed for 20 minutes in $4 \%$ paraformaldehyde, $3 \%$ triton X-10o in phosphate-buffered saline. Primary antibodies were rabbit anti-GFP (1:250) from Torres Pines Biolabs (Houston, TX, USA), or (1:500) from Molecular Probes (Invitrogen, Carlsbad, CA, USA), mouse anti-eys 21A6 and mouse anti-Futch 22C10 (kindly provided by S Benzer), mouse anti-elav 9F8A9 (1:500) obtained from the Developmental Studies Hybridoma Bank, Iowa City, IA, USA. Secondary conjugated antibodies were A488 and A546-anti-mouse and anti-rabbit (Molecular Probes, Invitrogen, Carlsbad, CA, USA). Images were obtained on a Zeiss Imager Z1 and LSM510 confocal microscope.

\section{Abbreviations}

BBS, Bardet Biedl syndrome; bp, base pair; CDS, coding sequence; DCBB, Drosophila cilia and basal body; IFT, intraflagellar transport; RFX, regulatory factor X; TRP, transient receptor potential.

\section{Authors' contributions}

A.L. and B.D. designed the experiments, wrote and revised completed this manuscript; A.L., E.E. and P.S. performed genome wide X boxes search. A.L., R.D. and G.G. realized the identification of the first set of target genes. A.L., R.B., V.R. and E.C. carried out fly genetic experiments. A.L., V.R. and R.B. performed cytology.

\section{Additional data files}

The following additional data are available with the online version of this paper. Additional data file 1 is a table listing the full set of 412 X-box genes conserved between $D$. melanogaster and $D$. pseudoobscura. Our X-box search across $D$. melanogaster and D. pseudoobscura species identified 412 genes with a conserved X-box both in sequence and distance upstream of the ATG of homologous genes between the two fly genomes. Additional data file 2 is a table of the DCBB genes list established for D. melanogaster. Genes presented in this table are homologous to proteins identified as putative or confirmed ciliary or basal body components. They are sorted as follows: a first group of genes with annotated molecular functions, a second group of genes for which homologs in vertebrates have been reported, a third group of genes with no vertebrate homolog. Each category is sorted by the number of studies reporting each gene or its homolog. Additional data file 1 is a table listing the number of Drosophila genes homologous to ciliary genes identified in previously published studies.

\section{Acknowledgements}

Work in the laboratory of BD was supported by the CNRS, the $\mathrm{ACl}$ "Jeune Chercheur" and $\mathrm{ACl}$ "Biologie du Développement" and by a Grant "ANR Maladies Rares" ( $n^{\circ} 930$ AR I7). R Dubruille was supported by a doctoral fellowship from the French Ministry of Education and Research and a fellowship from the Fondation pour la Recherche Médicale. G Grenier was supported by a doctoral fellowship from the Région Rhône-Alpes and the Fondation pour la Recherche Médicale. Work in the laboratory of PS was supported by the Swedish Research Council (VR) and by the Swedish Foundation for Strategic Research (SSF). Special thanks go to Stephen Richards and William Gilbert, who provided files prior to public release. We are indebted to Laurent Duret for precious help with genome comparisons and programming. We wish to thank Mélodie Robach, Maria Ouzounova and Abdelkader Selmi for their technical contribution during undergraduate internships and Jérome Schmitt for helpful technical assistance with the fly stocks. Confocal microscopy observations were performed at the CT $\mu$ of the Université Lyon I. We also wish to thank Joelle Thomas for careful reading of the manuscript. 


\section{References}

I. Rosenbaum JL, Witman GB: Intraflagellar transport. Nat Rev Mol Cell Biol 2002, 3:8I3-825.

2. Pan J, Wang Q, Snell WJ: Cilium-generated signaling and ciliarelated disorders. Lab lnvest 2005, 85:452-463.

3. Marshall WF, Nonaka S: Cilia: tuning in to the cell's antenna. Curr Biol 2006, 16:R604-6I4.

4. Singla V, Reiter JF: The primary cilium as the cell's antenna: signaling at a sensory organelle. Science 2006, 3 I 3:629-633.

5. Badano JL, Mitsuma N, Beales PL, Katsanis N: The ciliopathies: an emerging class of human genetic disorders. Annu Rev Genomics Hum Genet 2006, 7:125-148.

6. Snell WJ, Pan J, Wang Q: Cilia and flagella revealed: from flagellar assembly in Chlamydomonas to human obesity disorders. Cell 2004, I I 7:693-697.

7. Bisgrove BW, Yost HJ: The roles of cilia in developmental disorders and disease. Development 2006, I33:4|3|-4|43.

8. Rosenbaum J: Intraflagellar transport. Curr Biol 2002, I 2:RI25.

9. Sloboda RD: A healthy understanding of intraflagellar transport. Cell Motil Cytoskeleton 2002, 52:I-8.

10. Ostrowski LE, Blackburn K, Radde KM, Moyer MB, Schlatzer DM, Moseley A, Boucher RC: A proteomic analysis of human cilia: identification of novel components. Mol Cell Proteomics 2002, I:45 I-465.

I I. Pazour GJ, Agrin N, Leszyk J, Witman GB: Proteomic analysis of a eukaryotic cilium. J Cell Biol 2005, I 70: I03-I I3.

12. Broadhead R, Dawe HR, Farr H, Griffiths S, Hart SR, Portman N, Shaw MK, Ginger ML, Gaskell SJ, McKean PG, et al.: Flagellar motility is required for the viability of the bloodstream trypanosome. Nature 2006, 440:224-227.

13. Avidor-Reiss T, Maer AM, Koundakjian E, Polyanovsky A, Keil T, Subramaniam S, Zuker CS: Decoding cilia function: defining specialized genes required for compartmentalized cilia biogenesis. Cell 2004, I I 7:527-539.

14. Li JB, Gerdes JM, Haycraft CJ, Fan Y, Teslovich TM, May-Simera H, Li $\mathrm{H}$, Blacque $\mathrm{OE}$, Li L, Leitch CC, et al: Comparative genomics identifies a flagellar and basal body proteome that includes the BBS5 human disease gene. Cell 2004, I I 7:54I-552.

15. Stolc V, Samanta MP, Tongprasit W, Marshall WF: Genome-wide transcriptional analysis of flagellar regeneration in Chlamydomonas reinhardtii identifies orthologs of ciliary disease genes. Proc Natl Acad Sci USA 2005, 102:3703-3707.

16. Brody SL, Yan XH, Wuerffel MK, Song SK, Shapiro SD: Ciliogenesis and left-right axis defects in forkhead factor HFH-4-null mice. Am J Respir Cell Mol Biol 2000, 23:45-5I.

17. Gresh L, Fischer E, Reimann A, Tanguy M, Garbay S, Shao X, Hiesberger T, Fiette L, Igarashi P, Yaniv M, et al.: A transcriptional network in polycystic kidney disease. EMBO J 2004, 23: I657-I668.

18. Reith W, Herrero-Sanchez C, Kobr M, Silacci P, Berte C, Barras E, Fey S, Mach B: MHC class II regulatory factor RFX has a novel DNA binding domain and functionally independant dimerization domain. Genes Dev 1990, 4: I528-I540.

19. Gajiwala KS, Chen H, Cornille F, Roques BP, Reith W, Mach B, Burley SK: Structure of the winged-helix protein $h R F I$ reveals a new mode of DNA binding. Nature 2000, 403:916-92I.

20. Emery $P$, Durand B, Mach B, Reith W: RFX proteins, a novel family of DNA binding proteins conserved in the eukaryotic kingdom. Nucleic Acids Res 1996, 24:803-807.

21. Swoboda P, Adler HT, Thomas JH: The RFX-type transcription factor DAF- 19 regulates sensory neuron cilium formation in C. elegans. Mol Cell 2000, 5:4 I I-42I.

22. Vandaele C, Coulon-Bublex M, Couble P, Durand B: Drosophila regulatory factor $X$ is an embryonic type I sensory neuron marker also expressed in spermatids and in the brain of Drosophila. Mech Dev 2001, 103:159-162.

23. Dubruille R, Laurencon A, Vandaele C, Shishido E, Coulon-Bublex M, Swoboda $\mathrm{P}$, Couble $\mathrm{P}$, Kernan M, Durand B: Drosophila regulatory factor $X$ is necessary for ciliated sensory neuron differentiation. Development 2002, I 29:5487-5498.

24. Bonnafe E, Touka M, AitLounis A, Baas D, Barras E, Ucla C, Moreau A, Flamant F, Dubruille R, Couble $P$, et al.: The transcription factor RFX3 directs nodal cilium development and left-right asymmetry specification. Mol Cell Biol 2004, 24:44I7-4427.

25. Baas D, Meiniel A, Benadiba C, Bonnafe E, Meiniel O, Reith W, Durand B: A deficiency in RFX3 causes hydrocephalus associated with abnormal differentiation of ependymal cells. Eur J Neurosci 2006, 24:1020-1030.
26. Ait-Lounis A, Baas D, Barras E, Benadiba C, Charollais A, Nlend Nlend R, Liegeois D, Meda P, Durand B, Reith W: Novel function of the ciliogenic transcription factor RFX3 in development of the endocrine pancreas. Diabetes 2007, 56:950-959.

27. Liu Y, Pathak N, Kramer-Zucker A, Drummond IA: Notch signaling controls the differentiation of transporting epithelia and multiciliated cells in the zebrafish pronephros. Development 2007, I34: I I I-I I 22.

28. Reith W, Mach B: The bare lymphocyte syndrome and the regulation of MHC expression. Annu Rev Immunol 200 I, 1 9:33 I-373.

29. Blackshear PJ, Graves JP, Stumpo DJ, Cobos I, Rubenstein JL, Zeldin DC: Graded phenotypic response to partial and complete deficiency of a brain-specific transcript variant of the winged helix transcription factor RFX4. Development 2003, I30:4539-4552

30. Zarbalis K, May SR, Shen Y, Ekker M, Rubenstein JL, Peterson AS: A focused and efficient genetic screening strategy in the mouse: identification of mutations that disrupt cortical development. PLoS Biol 2004, 2:E219.

31. Araki R, Takahashi H, Fukumura R, Sun F, Umeda N, Sujino M, Inouye ST, Saito T, Abe M: Restricted expression and photic induction of a novel mouse regulatory factor $X 4$ transcript in the suprachiasmatic nucleus. I Biol Chem 2004, 279: I0237-10242.

32. Zhang D, Zeldin DC, Blackshear PJ: Regulatory factor X4 variant 3: A transcription factor involved in brain development and disease. J Neurosci Res 2007 in press.

33. Haycraft CJ, Schafer JC, Zhang Q, Taulman PD, Yoder BK: Identification of CHE-13, a novel intraflagellar transport protein required for cilia formation. Exp Cell Res 2003, 284:249-26I.

34. Haycraft CJ, Swoboda P, Taulman PD, Thomas JH, Yoder BK: The C. elegans homolog of the murine cystic kidney disease gene Tg737 functions in a ciliogenic pathway and is disrupted in osm-5 mutant worms. Development 200I, I 28: I 493-I505.

35. Schafer JA, Haycraft CJ, Thomas JH, Yoder BK, Swoboda P: xbx-I encodes a dynein light intermediate chain (DLIC) required for retrograde intraflagellar transport and cilia assembly in C. elegans. Mol Biol Cell 2003, I 4:2057-2070.

36. Efimenko E, Bubb K, Mak HY, Holzman T, Leroux MR, Ruvkun G, Thomas $\mathrm{JH}$, Swoboda P: Analysis of xbx genes in C. elegans. Development 2005, I32:1923-1934.

37. Blacque OE, Perens EA, Boroevich KA, Inglis PN, Li C, Warner A, Khattra J, Holt RA, Ou G, Mah AK, et al.: Functional genomics of the cilium, a sensory organelle. Curr Biol 2005, I 5:935-94 I.

38. Chen N, Mah A, Blacque OE, Chu J, Phgora K, Bakhoum MW, Hunt Newbury CR, Khattra J, Chan S, Go A, et al: Identification of ciliary and ciliopathy genes in Caenorhabditis elegans through comparative genomics. Genome Biol 2006, 7:R I26.

39. Fan Y, Esmail MA, Ansley SJ, Blacque OE, Boroevich K, Ross AJ Moore SJ, Badano JL, May-Simera H, Compton DS, et al.: Mutations in a member of the Ras superfamily of small GTP-binding proteins causes Bardet-Biedl syndrome. Nat Genet 2004, 36:989-993.

40. Richards S, Liu Y, Bettencourt BR, Hradecky P, Letovsky S, Nielsen R, Thornton K, Hubisz MJ, Chen R, Meisel RP, et al.: Comparative genome sequencing of Drosophila pseudoobscura: chromosomal, gene, and cis-element evolution. Genome Res 2005 , I5: $1-18$

41. Bergman CM, Pfeiffer BD, Rincon-Limas DE, Hoskins RA, Gnirke A, Mungall CJ, Wang AM, Kronmiller B, Pacleb J, Park S, et al.: Assessing the impact of comparative genomic sequence data on the functional annotation of the Drosophila genome. Genome Biol 2002, 3:RESEARCH0086.

42. Emberly E, Rajewsky N, Siggia ED: Conservation of regulatory elements between two species of Drosophila. BMC Bioinformatics 2003, 4:57.

43. Berman BP, Pfeiffer BD, Laverty TR, Salzberg SL, Rubin GM, Eisen MB, Celniker SE: Computational identification of developmental enhancers: conservation and function of transcription factor binding-site clusters in Drosophila melanogaster and Drosophila pseudoobscura. Genome Biol 2004, 5:R6I

44. Andersen JS, Wilkinson CJ, Mayor T, Mortensen P, Nigg EA, Mann M: Proteomic characterization of the human centrosome by protein correlation profiling. Nature 2003, 426:570-574.

45. Keller LC, Romijn EP, Zamora I, Yates JR 3rd, Marshall WF: Proteomic analysis of isolated Chlamydomonas centrioles reveals orthologs of ciliary-disease genes. Curr Biol 2005, I 5: I 090-1098.

46. Gherman A, Davis EE, Katsanis N: The ciliary proteome database: an integrated community resource for the genetic and 
functional dissection of cilia. Nat Genet 2006, 38:96I-962.

47. Inglis PN, Boroevich KA, Leroux MR: Piecing together a ciliome. Trends Genet 2006, 22:491-500.

48. The Ciliary Proteome Database [http://www.ciliapro teome.org/]

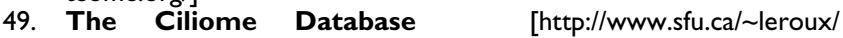
ciliome_database.htm]

50. Hou Y, Qin H, Follit JA, Pazour GJ, Rosenbaum JL, Witman GB: Functional analysis of an individual IFT protein: IFT46 is required for transport of outer dynein arms into flagella. J Cell Biol 2007, I 76:653-665.

5I. Bell LR, Stone S, Yochem J, Shaw JE, Herman RK: The molecular identities of the Caenorhabditis elegans intraflagellar transport genes dyf-6, daf-10 and osm-1. Genetics 2006, | 73: | 275- | 286.

52. Eberl DF, Hardy RW, Kernan MJ: Genetically similar transduction mechanisms for touch and hearing in Drosophila. J Neurosci 2000, 20:598I-5988.

53. Bahe S, Stierhof YD, Wilkinson CJ, Leiss F, Nigg EA: Rootletin forms centriole-associated filaments and functions in centrosome cohesion. J Cell Biol 2005, I 7 I:27-33.

54. Yang J, Liu X, Yue G, Adamian M, Bulgakov O, Li T: Rootletin, a novel coiled-coil protein, is a structural component of the ciliary rootlet. J Cell Biol 2002, I 59:43 I-440.

55. Yang J, Gao J, Adamian M, Wen XH, Pawlyk B, Zhang L, Sanderson MJ, Zuo J, Makino CL, Li T: The ciliary rootlet maintains longterm stability of sensory cilia. Mol Cell Biol 2005, 25:4I29-4I37.

56. Yang J, Adamian M, Li T: Rootletin interacts with C-Nap I and may function as a physical linker between the pair of centrioles/basal bodies in cells. Mol Biol Cell 2006, I 7: I033-1040.

57. Andrade YN, Fernandes J, Vazquez E, Fernandez-Fernandez JM, Arniges $M$, Sanchez TM, Villalon M, Valverde MA: TRPV4 channel is involved in the coupling of fluid viscosity changes to epithelial ciliary activity. J Cell Biol 2005, I 68:869-874.

58. Reiter JF, Skarnes WC: Tectonic, a novel regulator of the Hedgehog pathway required for both activation and inhibition. Genes Dev 2006, 20:22-27.

59. Baron DM, Ralston KS, Kabututu ZP, Hill KL: Functional genomics in Trypanosoma brucei identifies evolutionarily conserved components of motile flagella. J Cell Sci 2007, I 20:478-49I.

60. Niu Y, Murata T, Watanabe K, Kawakami K, Yoshimura A, Inoue J, Puri RK, Kobayashi N: MIP-T3 associates with IL-I 3Ralpha I and suppresses STAT6 activation in response to IL-I3 stimulation. FEBS Lett 2003, 550:|39-|43.

6I. Laoukili J, Perret E, Willems T, Minty A, Parthoens E, Houcine O, Coste A, Jorissen M, Marano F, Caput D, et al:: IL- I 3 alters mucociliary differentiation and ciliary beating of human respiratory epithelial cells. J Clin Invest 200I, I08:1817-1824.

62. Skowron M, Perret E, Marano F, Caput D, Tournier F: Interleukin13 alters mucociliary differentiation of human nasal epithelial cells. Chest 2003, I 23:373S-374S.

63. Takemaru K, Yamaguchi S, Lee YS, Zhang Y, Carthew RW, Moon RT: Chibby, a nuclear beta-catenin-associated antagonist of the Wnt/Wingless pathway. Nature 2003, 422:905-909.

64. Li FQ, Singh AM, Mofunanya A, Love D, Terada N, Moon RT, Takemaru K: Chibby promotes adipocyte differentiation through inhibition of \{beta\}-catenin signaling. Mol Cell Biol 2007, 27:4347-4354.

65. Singh AM, Li FQ, Hamazaki T, Kasahara H, Takemaru K, Terada N: Chibby, an antagonist of the Wnt/beta-catenin pathway, facilitates cardiomyocyte differentiation of murine embryonic stem cells. Circulation 2007, I 15:617-626.

66. The Berkeley Drosophila Genome Project [http://www.fruit fly.org/cgi-bin/ex/insitu.pl]

67. Uga S, Kuwabara M: On the fine structure of the chordotonal sensillum in antenna of Drosophila melanogaster. J Electron Microsc 1965, 14:173-181.

68. Moulins M: Ultrastructure of chordotonal organs. In Structure and Function of Proprioreceptors in the Invertebrates Edited by: Mill PJ. London: Chapman and Hall; 1976:387-426.

69. Husain N, Pellikka M, Hong H, Klimentova T, Choe KM, Clandinin TR, Tepass U: The agrin/perlecan-related protein eyes shut is essential for epithelial lumen formation in the Drosophila retina. Dev Cell 2006, I I:483-493.

70. Dawe HR, Smith UM, Cullinane AR, Gerrelli D, Cox P, Badano JL, Blair-Reid S, Sriram N, Katsanis N, Attie-Bitach T, et al.: The MeckelGruber Syndrome proteins MKSI and meckelin interact and are required for primary cilium formation. Hum Mol Genet
2007, 16:173-186.

7I. Markstein M, Levine M: Decoding cis-regulatory DNAs in the Drosophila genome. Curr Opin Genet Dev 2002, I 2:60 I-606.

72. Zhao G, Schriefer LA, Stormo GD: Identification of muscle-specific regulatory modules in Caenorhabditis elegans. Genome Res 2007, I 7:348-357.

73. Ray K, Perez SE, Yang Z, Xu J, Ritchings BW, Steller H, Goldstein LS: Kinesin-II is required for axonal transport of choline acetyltransferase in Drosophila. J Cell Biol 1999, 147:507-5I8.

74. Ou G, Koga M, Blacque OE, Murayama T, Ohshima Y, Schafer JC, Li C, Yoder BK, Leroux MR, Scholey JM: Sensory ciliogenesis in Caenorhabditis elegans: assignment of IFT components into distinct modules based on transport and phenotypic profiles. Mol Biol Cell 2007, I 8: I554-I569.

75. Basto R, Lau J, Vinogradova T, Gardiol A, Woods CG, Khodjakov A Raff JW: Flies without centrioles. Cell 2006, I 25: I375-I 386.

76. Peel N, Stevens NR, Basto R, Raff JW: Overexpressing centriolereplication proteins in vivo induces centriole overduplication and de novo formation. Curr Biol 2007, I 7:834-843.

77. Rodrigues-Martins A, Bettencourt-Dias M, Riparbelli M, Ferreira C, Ferreira I, Callaini G, Glover DM: DSAS-6 organizes a tube-like centriole precursor, and its absence suggests modularity in centriole assembly. Curr Biol 2007, I7:|465-|472.

78. Rodrigues-Martins A, Riparbelli M, Callaini G, Glover DM, Bettencourt-Dias M: Revisiting the role of the mother centriole in centriole biogenesis. Science 2007, 3 I 6: 1046-1050.

79. Gopfert MC, Robert D: Motion generation by Drosophila mechanosensory neurons. Proc Natl Acad Sci USA 2003, I 00:55 | 4-55 I 9

80. Moran DT, Varela FJ, Rowley JC 3rd: Evidence for active role of cilia in sensory transduction. Proc Natl Acad Sci USA 1977, 74:793-797.

81. Haycraft CJ, Banizs B, Aydin-Son Y, Zhang Q, Michaud EJ, Yoder BK: Gli2 and Gli3 localize to cilia and require the intraflagellar transport protein polaris for processing and function. PLOS Genet 2005, I :e53.

82. Huangfu D, Anderson KV: Signaling from Smo to Ci/Gli: conservation and divergence of Hedgehog pathways from Drosophila to vertebrates. Development 2006, I 33:3- I4.

83. Caspary T, Larkins CE, Anderson KV: The graded response to Sonic Hedgehog depends on cilia architecture. Dev Cell 2007, I 2:767-778.

84. Rebeiz M, Posakony JW: GenePalette: a universal software tool for genome sequence visualization and analysis. Dev Biol 2004, 271:431-438.

85. Celniker SE, Wheeler DA, Kronmiller B, Carlson JW, Halpern A, Patel S, Adams M, Champe M, Dugan SP, Frise E, et al.: Finishing a whole-genome shotgun: release 3 of the Drosophila melanogaster euchromatic genome sequence. Genome Biol 2002 3:RESEARCH0079.

86. VISTA [http://genome.lbl.gov/vista]

87. Marshall WF: Human cilia proteome contains homolog of zebrafish polycystic kidney disease gene qilin. Curr Biol 2004, |4:R9|3-9|4.

88. Loppin B, Lepetit D, Dorus S, Couble P, Karr TL: Origin and neofunctionalization of a Drosophila paternal effect gene essential for zygote viability. Curr Biol 2005, I 5:87-93.

89. Spradling AC: $\mathbf{P}$ element mediated transformation. In Drosophila: a Practical Approach Edited by: Roberts D. Oxford: IRL Press; 1986:175-189.

90. Rothwell WF, Sullivan W: Fluorescent analysis of Drosophila embryos. In Drosophila Protocols Edited by: Sullivan W, Ashburner M Hawley RS. Cold Spring Harbor, NY: Cold Spring Harbor Laboratory Press; 2000:|14|-I58.

91. Frazer KA, Pachter L, Poliakov A, Rubin EM, Dubchak I: VISTA: computational tools for comparative genomics. Nucleic Acids Res 2004, 32:W273-279.

92. Han $\mathrm{YG}$, Kwok $\mathrm{BH}$, Kernan MJ: Intraflagellar transport is required in Drosophila to differentiate sensory cilia but not sperm. Curr Biol 2003, I3:1679-1686.

93. Murayama T, Toh $Y$, Ohshima $Y$, Koga M: The dyf-3 gene encodes a novel protein required for sensory cilium formation in Caenorhabditis elegans. I Mol Biol 2005, 346:677-687.

94. Efimenko E, Blacque OE, Ou G, Haycraft CJ, Yoder BK, Scholey JM, Leroux MR, Swoboda P: Caenorhabditis elegans DYF-2, an orthologue of human WDRI9, is a component of the intraflagellar transport machinery in sensory cilia. Mol Biol Cell 2006, I 7:480I-48II.

95. Yu H, Pretot RF, Burglin TR, Sternberg PW: Distinct roles of tran- 
scription factors EGL-46 and DAF- 19 in specifying the functionality of a polycystin-expressing sensory neuron necessary for C. elegans male vulva location behavior. Development 2003, 130:5217-5227.

96. Winkelbauer ME, Schafer JC, Haycraft CJ, Swoboda P, Yoder BK: The $C$. elegans homologs of nephrocystin-I and nephrocystin-4 are cilia transition zone proteins involved in chemosensory perception. J Cell Sci 2005, I I 8:5575-5587. 